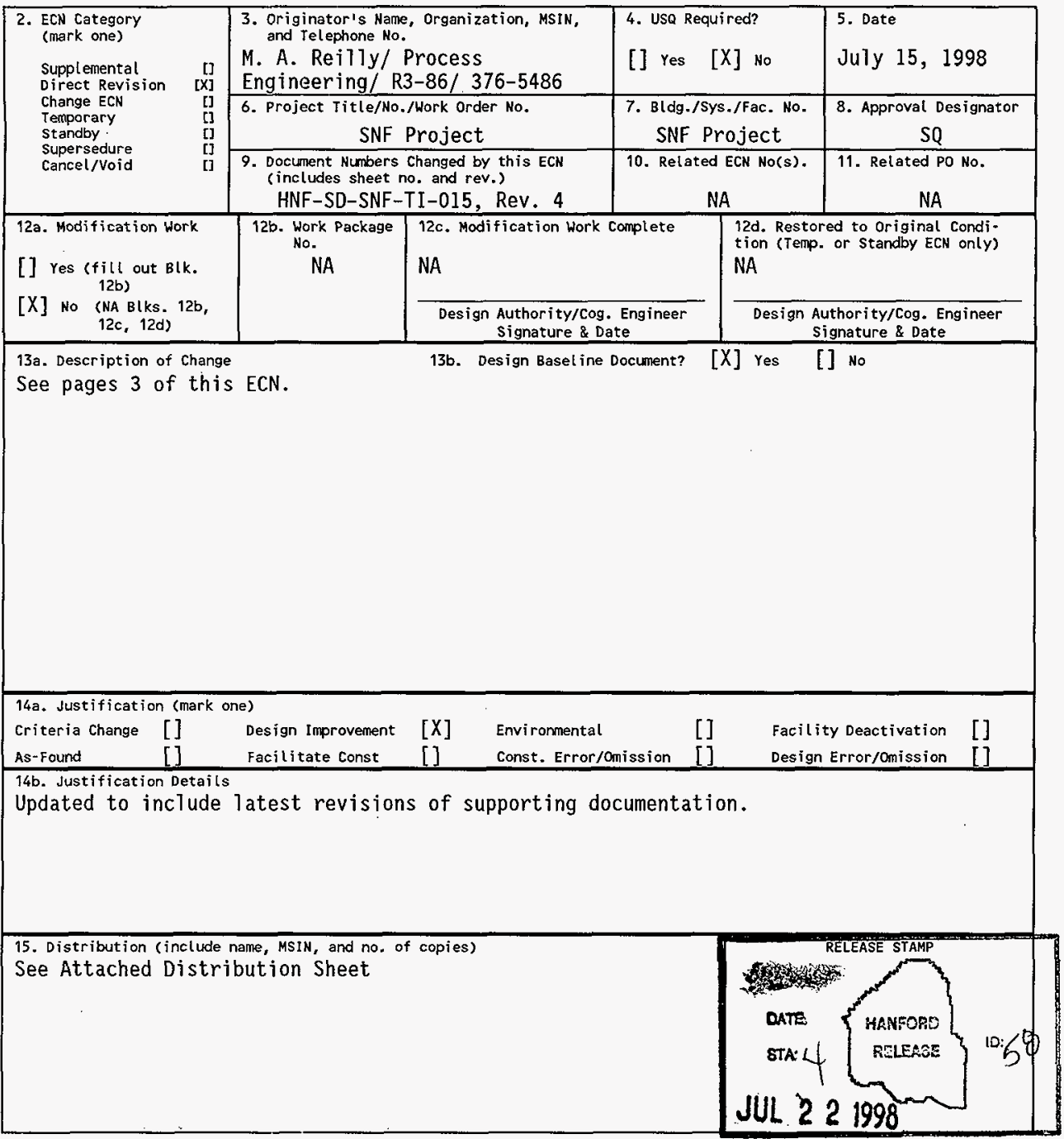




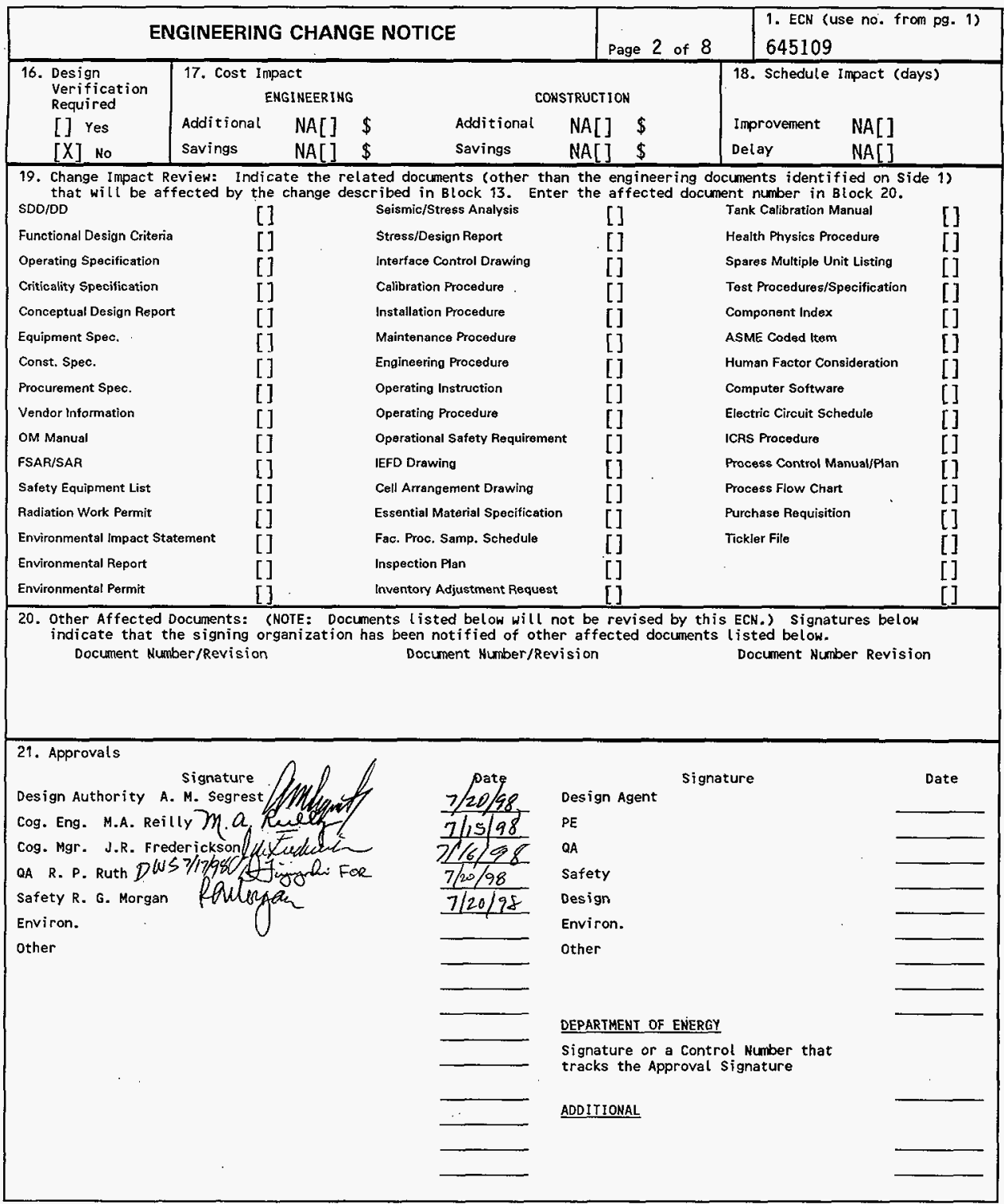




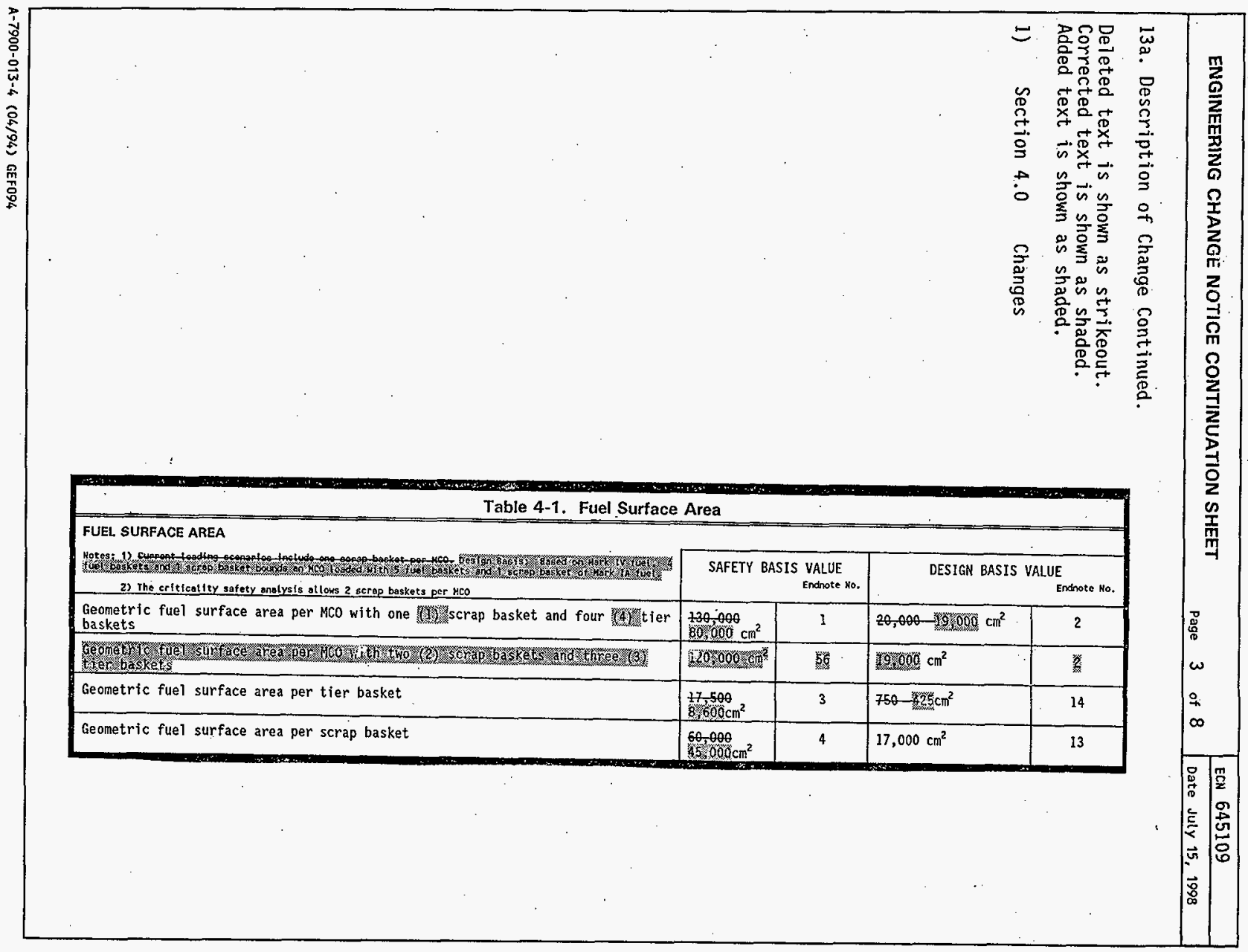


2) Section 5.0 Changes

Table 5-2. MCO Maximum Internal Pressure

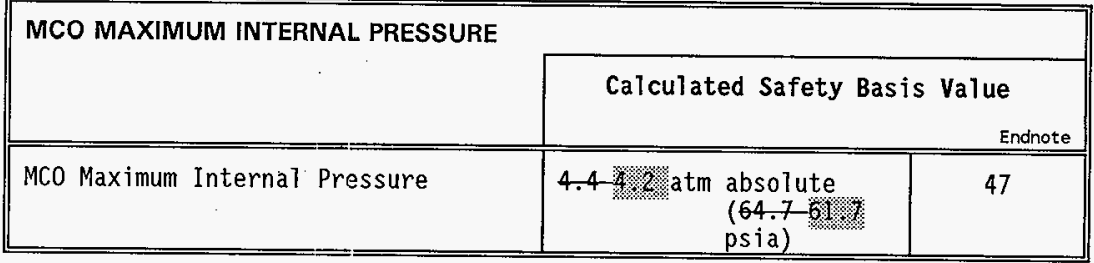

Table 5-3. MCO Maximum Temperatures in CSB Storage

\begin{tabular}{|c|c|c|}
\hline \multicolumn{3}{|l|}{ MCO MAXIMUM TEMPERATURES in CSB STORAGE } \\
\hline Honglov o & \multicolumn{2}{|c|}{ Calculated Safety Basis Value } \\
\hline & & Endnote \\
\hline MCO Maximum WaTl Temperature & $\left(226^{\circ} \mathrm{F}\right)$ & 48 \\
\hline MCO Maximum Gas Temperature & $\left(257^{\circ} \mathrm{F}\right)$ & 49 \\
\hline MCO Maximum Fuel Temi erature & $(28230 \% \div \%$ & 50 \\
\hline 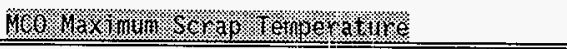 & 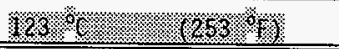 & 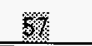 \\
\hline
\end{tabular}




\section{Page 5 of 8}

ECN 645109

Date July 15, 1998

4) Section 6.0 Changes

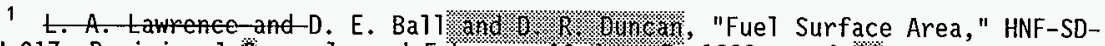
SNF-CN-017, Revision +-2, released Fertary 10 Jure 5, 1998, p. Z-10.

2 HNF-SD-SNF-CN-017, Revision 1-2, released February 10-Jine 5,1998 , p. 5-7.

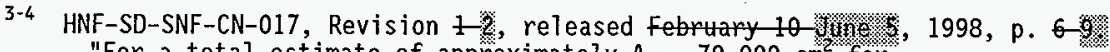
"For a total estimate of approximate $7 y A_{\bar{g}}-70,000 \mathrm{~cm}-$ for taged fuet."

$70,000 \mathrm{~cm}^{2}-4$ fuel baskets $-17,500 \mathrm{~cm}^{2}$

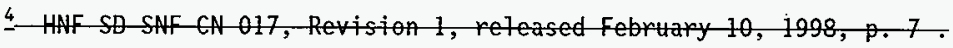

10-12 A. L. Pajunen, "Evaluation of Radiolytic Gas Generation from Water

Dissociation in a Multi-Canister Overpack, "HNF-SD-SNF-CN-006, Revision 0, released Apri1 21, 1996-\%, p. 10, Table 1.

$13-14$ HNF-SD-SNF-CN-017, Revision I-2, released Fy 10 10 5 \%.

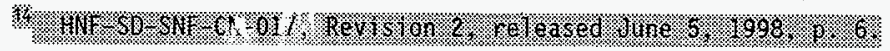

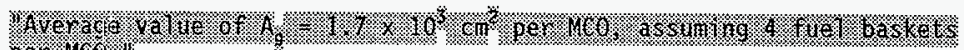
10. $116 \%$ :

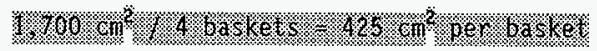

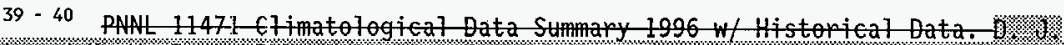

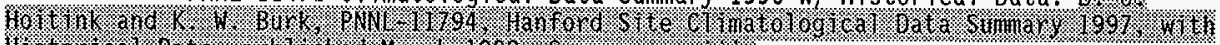

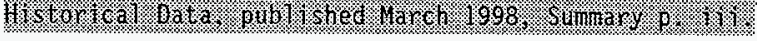

47 J. R. Frederickson and M. G. Plys, "MCO Internal Gas Composition and Pressure During Interim Storage," HNF-SD-SNF-TI-040, Revision 1-2, released Harch 19-3ime I8, 1998 , p. Z - 3-2.

48 - 49 D. R. Duncan and M. G. Plys, "Simulation of Normal and Off-Normal MultiCanister Overpack Behavior," HNF-2256, Revision $\theta-1$, released April 9-1161\% I, 1998, p. $+127-173$.

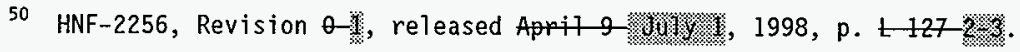

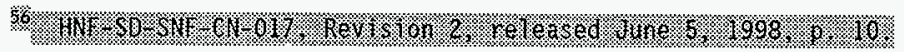

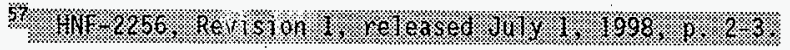


Table 6-1. Technical Databook Supporting Document RevieW Status

\begin{tabular}{|c|c|c|c|c|c|c|}
\hline Document Number & Document Title & Author & 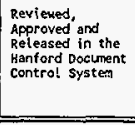 & 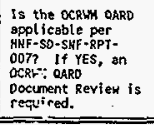 & 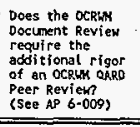 & 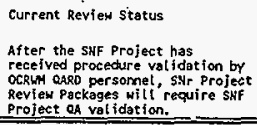 \\
\hline $\begin{array}{l}\text { HNF-2586, } \\
\text { Revision } 0\end{array}$ & $\begin{array}{l}\text { Summary Assessment of } \\
\text { Fuel Damage } \\
\text { Distributions in the } K \\
\text { Basins }\end{array}$ & A. L. Pitner & $4 / 23 / 98$ & YES & YES & $\begin{array}{l}\text { Review Required in } \\
\text { accordance with } \\
\text { AP 6-009. }\end{array}$ \\
\hline $\begin{array}{l}\text { HNF-SD-SNF-TI-009, } \\
\text { Revision } 1\end{array}$ & $\begin{array}{l}\text { 105-K Basin Material } \\
\text { Design Basis -Feed } \\
\text { Description for SNF } \\
\text { Project Facilities }\end{array}$ & $\begin{array}{l}\text { W. L. Will is and } \\
\text { A. N. Praga : }\end{array}$ & $1 / 9 / 98$ & YES & YES & $\begin{array}{l}\text { Review Required in } \\
\text { accordance with } \\
\text { AP 6-009. }\end{array}$ \\
\hline $\begin{array}{l}\text { WHC-SD-SNF-TI-016, } \\
\text { Revision OD and } \\
\text { stpptementat ECH } \\
\text { 638939 }\end{array}$ & $\begin{array}{l}\text { Development of Design } \\
\text { Basis Capacity for SNF } \\
\text { Project Systems }\end{array}$ & A. L. Pajunen & $\begin{array}{l}2 / 27 / 96 \text {-and } \\
\qquad C M-1 / 21 / 97 \\
7 / 27 / 9 \%\end{array}$ & No & $\begin{array}{l}\text { Not } \\
\text { Applicable }\end{array}$ & Not App] icable \\
\hline $\begin{array}{l}\text { WhC-SD-SNF-ANAL-01I } \\
\text { Revision } 0 .\end{array}$ & $\begin{array}{l}\text { Comparison of Spent } \\
\text { Fuel Databases for } K \\
\text { Basins }\end{array}$ & $\begin{array}{l}\text { F. Schmittroth \& } \\
\text { K. D. Dobbin }\end{array}$ & $9 / 12 / 96$ & no & $\begin{array}{l}\text { Not } \\
\text { Applicable }\end{array}$ & Not Applicable \\
\hline $\begin{array}{l}\text { Thermophysical } \\
\text { Properties of Matter } \\
\text { Volume } 7\end{array}$ & $\begin{array}{l}\text { Thermal Radiative } \\
\text { Properties, Metallic } \\
\text { Elements and Atloys }\end{array}$ & $\begin{array}{l}\text { Thermophysical } \\
\text { Properties } \\
\text { Research Center }\end{array}$ & $\begin{array}{l}\text { Published } \\
1970\end{array}$ & NO & $\begin{array}{l}\text { Not } \\
\text { Applicable }\end{array}$ & Not Applicable \\
\hline 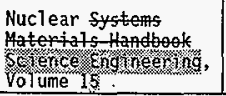 & $\begin{array}{l}\text { Determinations of the } \\
\text { Total Emissivity of } \\
\text { Polished Oxidized } \\
\text { Uranium Surfaces }\end{array}$ & $\begin{array}{l}\text { L. Baker Jr., et } \\
\text { ai. }\end{array}$ & $\begin{array}{l}\text { Published } \\
1963\end{array}$ & NO & $\begin{array}{l}\text { Not } \\
\text { Applicable }\end{array}$ & Not Applicabie \\
\hline
\end{tabular}




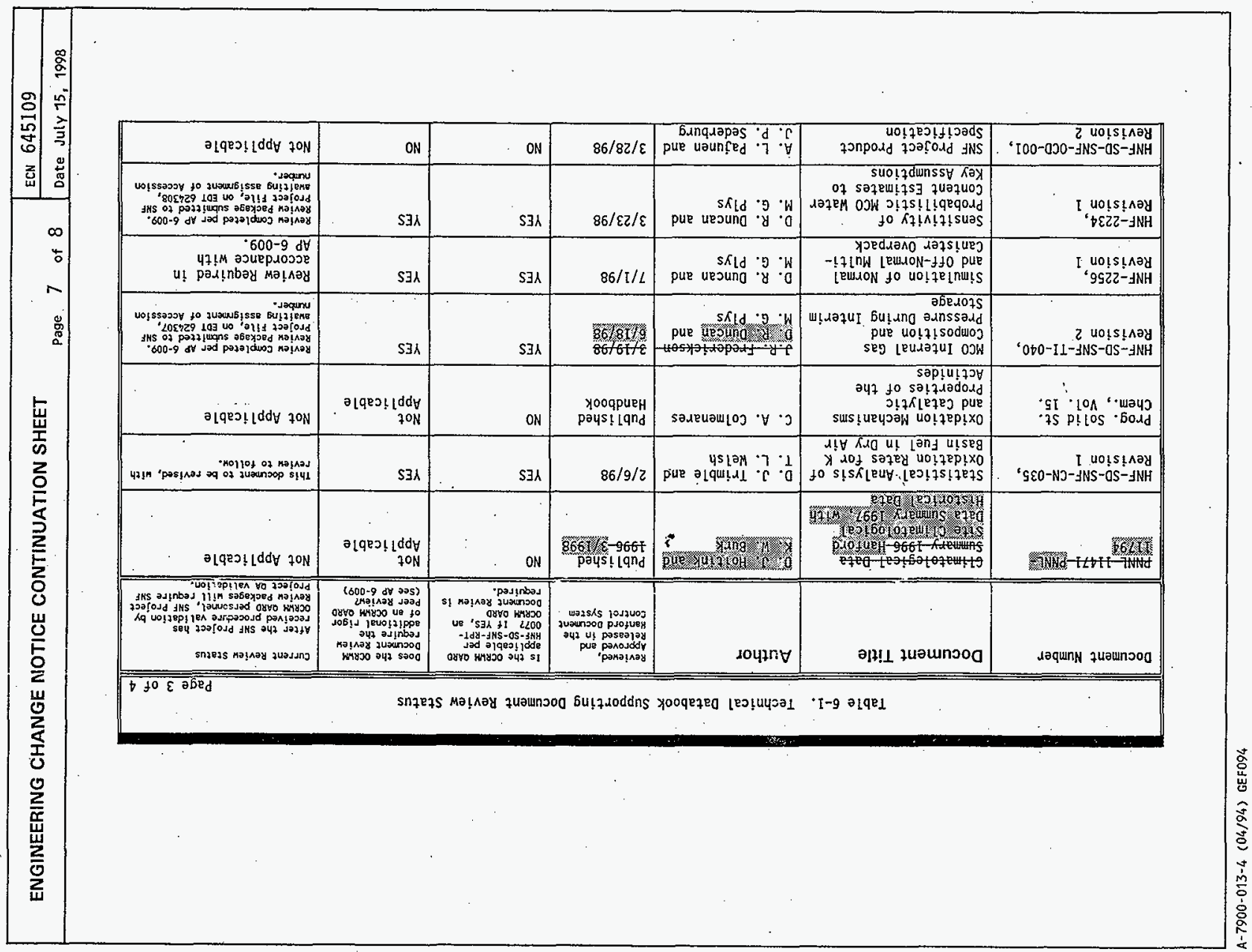


Table 6-1. Technical Databook Supporting Document Review Status

Page 1 . of 4

\begin{tabular}{|c|c|c|c|c|c|c|}
\hline Document Number & Document Title & Author & $\begin{array}{l}\text { Reviewed, } \\
\text { Approved and } \\
\text { Released in the } \\
\text { Hanford Document } \\
\text { Contro! syst: }\end{array}$ & 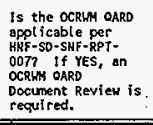 & $\begin{array}{l}\text { Does the OCRLw } \\
\text { Docunent Review } \\
\text { require the } \\
\text { additional rigor } \\
\text { of an oCRIN } 2 \text { \&Ro } \\
\text { Peer Review? } \\
\text { (See AP } 6-009 \text { ) } \\
\end{array}$ & 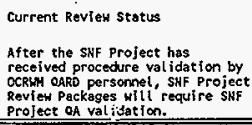 \\
\hline $\begin{array}{l}\text { HNF-SD-SNF-CN-017, } \\
\text { Revision } 1-2\end{array}$ & $\begin{array}{c}\text { Fuel Surface Area } \\
\therefore\end{array}$ & 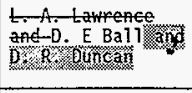 & $\frac{2+10 / 98}{6 \% 598}$ & YES & YES & $\begin{array}{l}\text { Revision } 2 \text { is being } \\
\text { Peer Reviewed in } \\
\text { accordance with AP } \\
6-009 \text {. }\end{array}$ \\
\hline $\begin{array}{l}\text { HNF-SD-SNF-TI-020, } \\
\text { Revision } 2 \\
\end{array}$ & $\begin{array}{l}\text { SNF Project Recommended } \\
\text { Reaction Rate Constants } \\
\text { for Corrosion of N- } \\
\text { Reactor Fuel }\end{array}$ & $\begin{array}{l}\text { T. D. Cooper, and } \\
\text { A. L. Pajunen : }\end{array}$ & $6 / 15 / 98$ & YES & YES & $\begin{array}{l}\text { Revien qackage in SNF project } \\
\text { file Acession, \# s29702316. } \\
\text { Review done prior to AP } \\
6-009 \text {, pkg may require } \\
\text { supplementation. }\end{array}$ \\
\hline $\begin{array}{l}\text { HNF-SD-W441-CN-001, } \\
\text { Revision } 1-3\end{array}$ & $\begin{array}{l}\text { SNF Inventory in Bulk } \\
\text { MCO Water at the Cold } \\
\text { Vacuum Drying Facility }\end{array}$ & D. L. Sherre 11 & $6 / 11 / 98$ & $\begin{array}{l}\text { To be } \\
\text { evaluated }\end{array}$ & $\begin{array}{l}\text { To be } \\
\text { evaluated }\end{array}$ & To be evaluated \\
\hline $\begin{array}{l}\text { HNF-SD-SNF-CN-006, } \\
\text { Revision } 0^{\circ}\end{array}$ & $\begin{array}{l}\text { Evaluation of } \\
\text { Radiolytic Gas } \\
\text { Generation from Water } \\
\text { Dissociation in a } \\
\text { Multi-Canister overpack }\end{array}$ & A. L. Pajunen & $4 / 21 / 96-3$ & YES & YES & $\begin{array}{l}\text { Revien Peckage in swF project } \\
\text { fite, Aecession \# s2970130. } \\
\text { Review done prior to AP } \\
6-009 \text {, pkg may require } \\
\text { supplementation. }\end{array}$ \\
\hline $\begin{array}{l}\text { HNF-1523, } \\
\text { Revision } 0\end{array}$ & $\begin{array}{l}\text { K Basins Particulate } \\
\text { Water Content, } \\
\text { Behavior, and Impact }\end{array}$ & $\begin{array}{l}\text { D. R. Duncan and } \\
\text { D. E. Ball }\end{array}$ & $11 / 19 / 98-7 / 2$ & YES & YES & $\begin{array}{l}\text { Review Completed per AP 6-009. } \\
\text { Review Package in SNF Project } \\
\text { File, Accession \# S2980602. }\end{array}$ \\
\hline $\begin{array}{l}\text { HNF-1527, } \\
\text { Revision } 0\end{array}$ & $\begin{array}{l}\text { Estimates of } \\
\text { Particulate Mass in } \\
\text { Multi-Canister } \\
\text { Overpacks } \\
\end{array}$ & $\begin{array}{l}\text { J. P. Sioughter, } \\
\text { et ai. }\end{array}$ & $11 / 19 / 97$ & YES & YES & $\begin{array}{l}\text { Revien Completed per AP 6-009. } \\
\text { ReYiew Pockage in SNF Project } \\
\text { File, Accession \# S2980602. }\end{array}$ \\
\hline
\end{tabular}




\title{
SPENT NUCLEAR FUEL PROJECT TECHNICAL DATABOOK
}

\author{
M. A. Reilly
}

DE\&S Hanford, Richland, WA 99352

U.S. Department of Energy Contract DE-AC06-96RL13200

$$
645109
$$

EDT/ECN: $645102 \mathrm{Ex}_{22} / \mathrm{sg}$

Org Code: $2 \mathrm{~T} 970$

UC: 510

B\&R Code: EW3135040

Charge Code: LBaSl

Total Pages: 38

Key Words: $105 \mathrm{~K}$ East Basin, $105 \mathrm{~K}$ West Basin, Canister Storage Building, Fuel Parameters, Siudge Parameters, Design Basis, Safety Basis, Vacuum Drying, Hot Conditioning, Fue] Movement, MCO, SPR

Abstract: The Spent Nuclear Fuet (SNF) Project Technical Databook is developed for use as a common authoritative source of fuel behavior and material parameters in support of the Hanford SNF Project. The Technical Databook will be revised as necessary to add parameters as their Databook submittals become available.

TRADEMARK DISCLAIMER. Reference herein to any specific comercial product, process, or service by trade name, trademark, manufacturer, or otherwise, does not necessarily constitute or imply its endorsement, recomendation, or favoring by the United states Government or any agency thereof or its contractors or subcontractors.

Printed in the United States of America. To obtain copies of this document, contact: Document Control Services, P.O. Box 950, Mailstop H6-08, Richland WA 99352, Phone (509) 372-2420;

Fax (509) 376-4989.
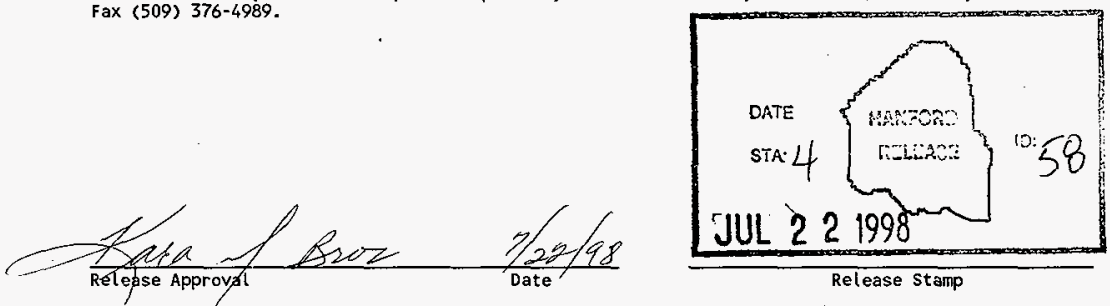

Approved for Public Release 
(2) Title

SPENT NUCLEAR FUEL PROJECT TECHNICAL DATABOOK

CHANGE CONTROL RECORD

Page 1 of 3

\begin{tabular}{|c|c|c|c|c|}
\hline \multirow{2}{*}{ (3) } & \multirow{2}{*}{ Revision } & \multirow{2}{*}{ (4) Description of Change - Replace, Add, and Delete Pages } & \multicolumn{2}{|c|}{ Authorized for Release } \\
\hline & & & (5) Cog. Engr. & (6) Cog. Mgr. \\
\hline & 0 & (7) Initial Release, EDT $613013,9 / 20 / 95$ & S. M. Scott & P. K. Shen $9 / 7 / 95$ \\
\hline & 1 & $\begin{array}{l}\text { Rev } 0 \text { with ECN } 191395 \text { completely rewritten } \\
\text { and reformatted, ECN } 638911,3 / 5 / 97\end{array}$ & D. R. Duncan & $\begin{array}{l}\text { J. R. Frederickson } \\
3 / 3 / 97\end{array}$ \\
\hline & 2 & $\begin{array}{l}\text { ECN } 638928 \\
\text { Section } 4 \text { and } 5 \text { reformatted. } \\
\text { Tables } 4-1,4-2,4-3,4-4,4-6,4-7,4-9 \text {, } \\
4-11,4-12 \text {, and } 4-13 \text { had parameters or } \\
\text { source document changes. }\end{array}$ & D. R. Duncan & $\begin{array}{l}\text { J. R. Frederickson } \\
\text { 5/1/97 }\end{array}$ \\
\hline & 3 & 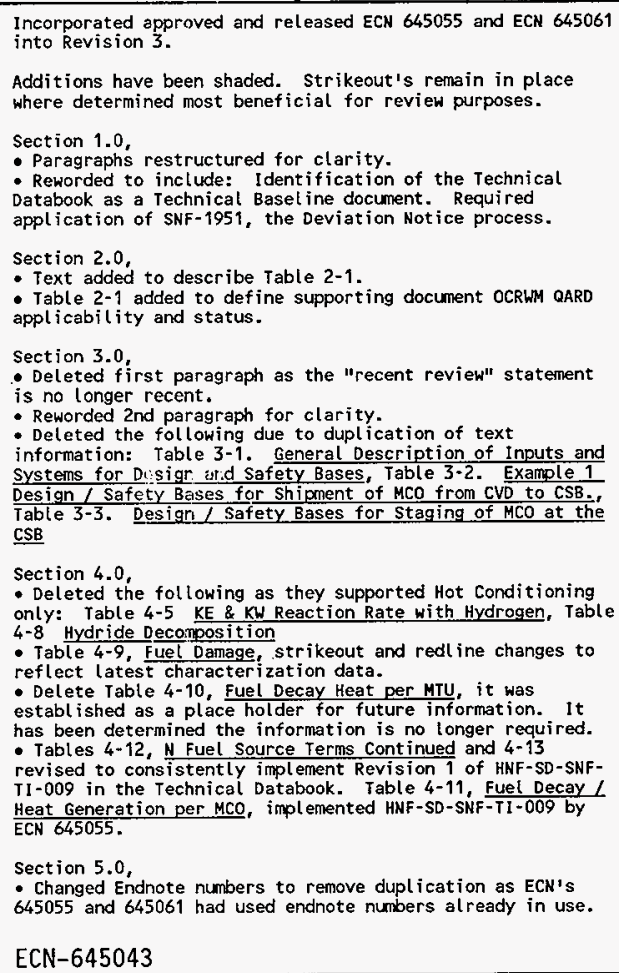 & $\begin{array}{l}\text { M. A. Reilly } \\
5 / 21 / 98\end{array}$ & $\begin{array}{l}\text { J. R. Frederickson } \\
5 / 21 / 98\end{array}$ \\
\hline
\end{tabular}


(3) Revision (4) Description of Change - Replace, Add, and Delete Pages

General changes:
- Rev 4 issued as Volume 1 of the Technical Databook.

- Sections 1,2 and 3: Deleted text not shown.

New text is identified by left margin vertical

lines.

Text relocated in the document is not redi ined.

Section 1, Paragraph 2 identifies Volume 1 pertaining to $k$ Basin Fuel, and volume 2 will address sludge and water

removal projects.

Section 2, Rewritten from describing sections of the databook, to stating how the values should be used within project analyses and modeling.

Section 3, Rewritten from a general discussion on design and safety analysis, to a definition section. Definitions specific to Technical Databook use.

Section 4, 1st, 2nd and 3rd paragraphs rewritten to

el iminate dupl ication of definitions now located in section 3. Values thit were changed are marked by strikeout and vertical lines in the left margin.

Table 4-7.

1. Corrected the endnotes to recognize the originating document for particulate mass numbers as HNF-1527, revision 0 .

2. Added Water Mass parameter and value for uncleaned fuel, endnote HNF-SD-SNF-OCD-001, revision 2.

3. Added value for residual free water estimate after cold vacuum drying, reference HNF-1851, revision 1.

Table 4-7a. Deleted, similar information presented as Table 5-1.

Section 5.0, Added text describing Derived Physical Quantities for calculated values, and why they are provided. Section includes tables that include calculation values, and endnotes (HNF-SD-W441-CN-001, revision 2; HNF-SD-SNF-Ti-040, revision 1; HNF-2256, revision 0; and HNF-2234, revision 1 .

Added: Table 5-1 Particulate Mass Generated per MCO Table 5-2 MCO Maximum Internal Pressure

Table 5-3 MCO Maximum Temperatures in CSB Storage Table 5-4 Water content associated with

Particulate in $\mathrm{MCO}$

Section 6.0 , Changes ta endnotes are indicated by strikeouts and left margin vertical lines.

Table 6-1 (had been Table 2-1) co-located with endnotes for convenience. Char jes are indicated by strikeouts and left margin vertical lines.

ECN-645102
Authorized for Release

(5) Cog. Engr. $(6)$ Cog. Mgr. Date

M. A. Reilly $6 / 18 / 98$

J. R. Frederickson $6 / 18 / 98$ 


\begin{tabular}{|c|c|c|c|c|}
\hline & \multirow{2}{*}{ Revision } & \multirow{2}{*}{ (4) Description of Change - Replace, Add, and Delete Pages } & \multicolumn{2}{|c|}{ Authorized for Release } \\
\hline & & & (5) Cog. Engr. & (6) Cog Mgr. \\
\hline R9 & 5 & $\begin{array}{l}\text { Changes are identified with a change bar in } \\
\text { the left margin of the document. } \\
\text { ECN-645109 shows corrected and additional } \\
\text { text as shaded, and deleted text as } \\
\text { strikeout. } \\
\text { Table } 4-1 \text {, Changed to reflect the analyses } \\
\text { completed in HNF-SD-SNF-CN-017, Revision } 2 \\
\text { (See Page } 3 \text { of ECN } 645109 \text { ). } \\
\text { Table 5-2, Changed to reflect the analyses } \\
\text { completed in HNF-SD-SNF-TI-040, Revision } 2 \\
\text { (See page } 4 \text { of ECN } 645109 \text { ). } \\
\text { Table 5-3, Changed to reflect the analyses } \\
\text { completed in HNF-2256, Revision } 1 \text { (See page } \\
4 \text { of ECN 645109). } \\
\text { Section 6.0, endnotes updated to source } \\
\text { documents for Table } 4-1 \text {, 5-2, and } 5-3 \\
\text { changes. Corrected editorial errors. (See } \\
\text { page } 5 \text { of ECN 645109). } \\
\text { Table 6-1, Corrected editorial errors, and } \\
\text { updated to current revisions of source } \\
\text { documents (See pages } 6-8 \text { of ECN } 645109 \text { ). }\end{array}$ & 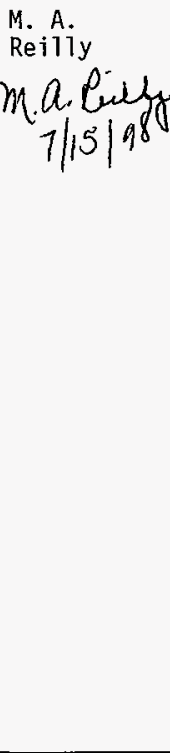 & $\begin{array}{l}\text { J. R. Notidewat } \\
\text { Frederickson } 7(6) / 86\end{array}$ \\
\hline & & & & \\
\hline & & & & \\
\hline & & & & \\
\hline & & & & \\
\hline & & & & \\
\hline & & & & \\
\hline
\end{tabular}


HNF-SD-SNF-TI-015, Revision 5

Table of Contents

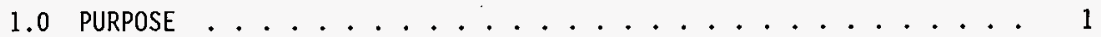

$2.0 \mathrm{SCOPE} \ldots \ldots \ldots \ldots \ldots \ldots$

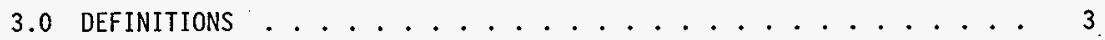

4.0 PARAMETER SUMMARY TABLE $\ldots \ldots \ldots \ldots$

5.0 DERIVED PHYSICAL QUANTITIES (calculated values) . . . . . . 24

6.0 ENDNOTES ............................... 26

List of Tables

Table 4-1. Fuel Surface Area .................. 4

Table 4-2. KE \& KW Fuel Reaction Enhancement Factor ......... 4

Table 4-3. KE \& KW Fuel Reaction Rate with Air.............. 5

Table 4-4. KE \& KW Reaction Rate with Water ............ 7

Table 4-5. Deleted .................... 8

Table 4-6. Radiolytic G VaTue ............. 8

Table 4-7. Canister Particulate Mass \& Water Content ........ 9

Table 4-7a. Deleted, See Section 5.0, Table 5-1.......... 11

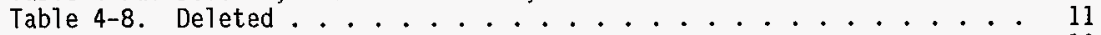

Table 4-9. Fuel Damage . . . . . . . . . . . . . 12

Table 4-10. Deleted . . . . . . . . . . . . . . 13

Table 4-11. Fuel Decay / Heat Generation per MCO . . . . . . . . 13

Table 4-12. N Fuel Source Terms . . . . . . . . . . . . . . 14

Table 4-13. Single Pass Reactor Source Terms ............ 21

Table 4-14. Thermal Emissivity . . . . . . . . . . . . . 23

Table 4-15. CSB Annual Temperature Cycle ............ 23

Table 5-1. Particulate Mass Generated per MCO . . . . . . . . 24

Table 5-2. MCO Maximum Internal Pressure ............ 25

Table 5-3. MCO Maximum Temperatures in CSB Storage ........ 25

Table 5-4. Water Content Associated with Particulate in MCO ..... 25

Table 6-1. Technical Databook Supporting Document Review Status .... 30

\section{List of Figures}

Figure 1. A Graphical Depiction of the SNF Project Technical Databook Sources and Receiving Products ............. 2 


\author{
HNF-SD-SNF-TI-015, Revision 5 \\ Spent Nuclear Fuel Project \\ Technical Databook \\ Volume 1, Fuel
}

\title{
1.0 PURPOSE
}

The Spent Nuclear Fuel (SNF) Project Technical Databook provides

project-approved summary tables of selected parameters and derived physical quantities, with nominal design and safety basis values. It contains the parameters necessary for a complete documentation basis of the SNF Project technical and safety baseline.

The databook is presented in two volumes. Volume 1 presents $K$ Basins SNF related information. Volume 2 (not yet available) will present selected sludge and water information, as it relates to the sludge and water removal projects.

\subsection{SCOPE}

The values, within this databook, shall be used as the foundation for analyses, modeling, assumptions, or other input to. SNF project safety analyses or design. All analysis and modeling using a parameter available in this databook are required to use and cite the appropriate associated value, and document any changes to those values (i.e., analys is assumptions, equipment conditions, etc). Characterization and analysis efforts are ongoing to validate, or update these values.

Figure 1 graphically depicts sources and receiving products of the Technical Databook. Sources include literature data, Characterization Program data, and analyses or calculations.

As a SNF Project Technical Baseline document, changes to this databook shall be controlled by both the Engineering Document Change Control

Requirements, HNF-PRO-440, and the SNF Baseline Change Contro7, HNF-1951. Before implementing any Technical Databook Engineering Change Notice (ECN), possible sub-project cost, scope and schedule impacts are to be evaluated per SNF -1951 . 
HNF-SD-SNF-TI-015, Revision 5

Figure 1. A Graphical Depiction of the SNF Project Technical Databook Sources and Receiving Products

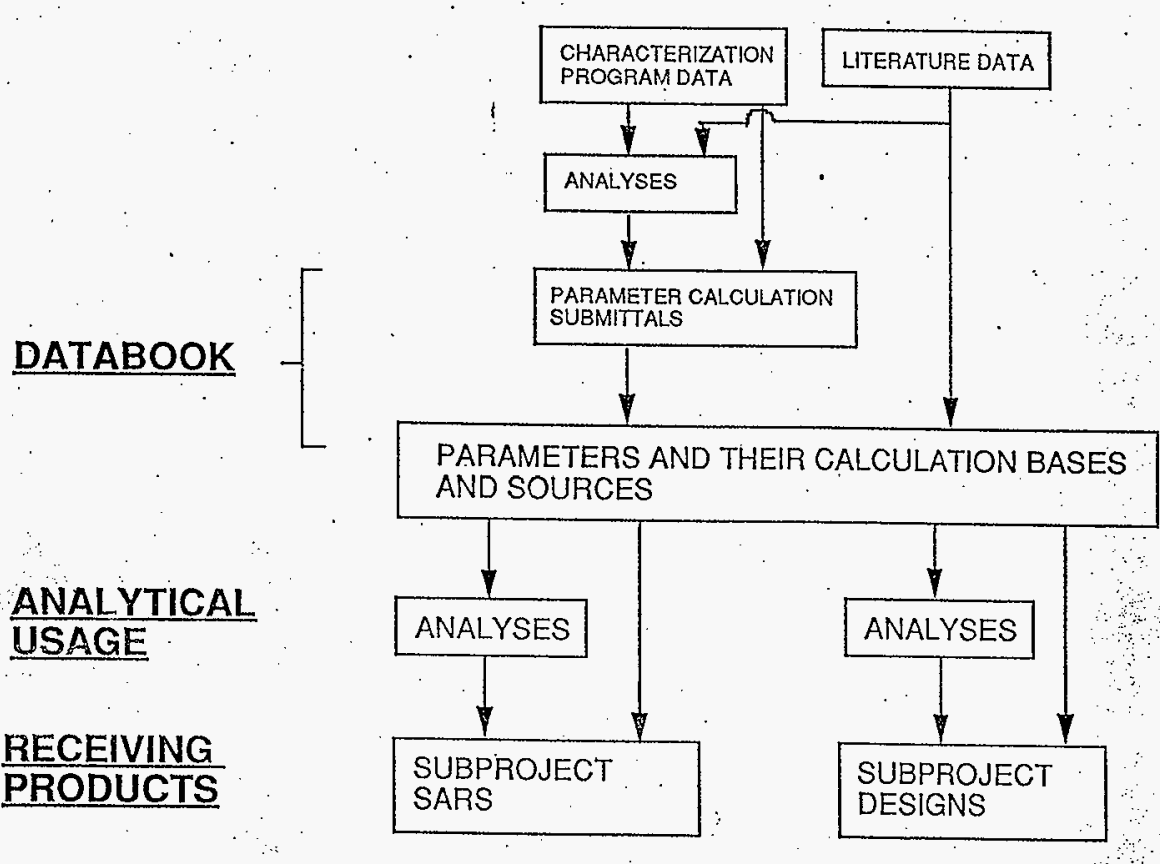


HNF-SD-SNF-TI-015, Revision 5

\subsection{DEFINITIONS}

design basis

The set of requirements that bound the design of structures, systems, or components within the facility. These design requirements include consideration of safety, plant availability, efficiency, reliability, and maintainability. Certain aspects of the design basis are important to

safety. (Basis: Project Hanford Management system (PHMS) Glossary)

design basis value

A nominal design basis value is based on anticipated operating conditions. A value range may exist, the conservative end of the performance range is used, whether the conservative end is high or low. (Basis: Technical Databook definition)

safety basis

The combination of information relating to the control of hazards at a nuclear facility (including design, engineering analyses, and administrative controls) upon which Department of Energy depends for its conclusions that activities at the facility can be conducted safely.

(Basis: PHMS Glossary)

safety basis value

A safety basis value is based on credible extremes of the parameter range. (Basis: Technical Databrok definition)

\subsection{PARAMETER SUMMARY TABLE}

The tables within this section present the key engineering, physical, and chemical parameters, with project-approved values. Included are basic $K$ Basin fuel physical properties, behavioral characteristics, and corrosion products, as well as parameters with interpreted values from technical handbooks and open Titerature.

Parameter values are given for both design basis and safety basis, according to the definitions in Section 3.0 . 


\begin{tabular}{|c|c|c|c|}
\hline 9I & $\varepsilon$ & s & OI \\
\hline 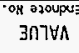 & & 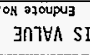 & \\
\hline
\end{tabular}

Ssauybnod of anp aspadou! pade aวefdns ant aut $\$ 1 \mathrm{~s}$

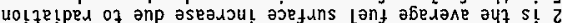

(s) $(2)=s$

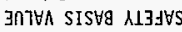

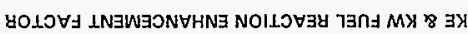

\section{0e}

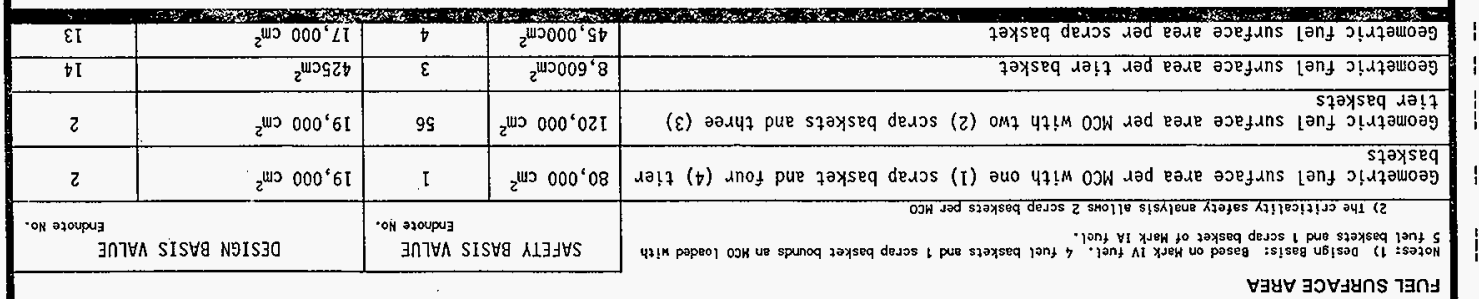


Table 4-3. KE \& KW Fuel Reaction Rate with Air

KE \& KW FUEL REACTION RATE (with air as a function of temperature, pressure, \& humidity)

Basic Rate Equation:

Rate [mg(ht gain)/hr] $=A_{0} \xi K$

Aranium, $\mathrm{cm}^{2}$,

$K=$ Rate Constant (can be dependent on temperature and reactant concentration), mg(ut gain)/hr-cm ${ }^{2}$

SAFETY BASIS VALUE

Rate

Constant

(K)

Units of $\mathrm{mg}$ (weight gain) $/ \mathrm{cm}^{2} / \mathrm{hr}$ where $P$ is the partial water pressure in $\mathrm{KPa}$,

$T$ is in $K$, and $R H$ is relative humidity.

$\frac{\text { Dry Air }\left(<10-15 \text { vppm } \mathrm{H}_{2} \text { ㅇ) }\right.}{U+\left(\frac{2+x}{2}\right) \mathrm{O}_{2} \rightarrow U \mathrm{O}_{2+x}}$

$$
\text { ...... Ut }\left(\frac{2+x}{2}\right) O_{2} \rightarrow U O_{2+x}
$$

for $T<692^{\circ} \mathrm{K} \quad \log K=7.19-3732 / \mathrm{T}$

for $T>692^{\circ} K \quad \log K=28.381-7 \log [T]-4638.2 / T$

Where $x<0.25$

Moist Air

$U+\left(\frac{2+x}{2}\right) O_{2} \rightarrow U O_{2 \cdot x}$

Where $x<0.25$

for $T<373 K(11-75 \% R H) \log K=13.8808-5769$
for $T<373 K(100 \% R H) \log K=8.333-3730 / T$

for $T<373 K(100 \% R H)$ Log $K=8.333-3730 / T$
for $373<T<463 K(<100 \% R H) \quad \operatorname{LogK}=10.566-4990 / T+0.3 \log [P]$

for $T>463 K(<100 \% R H) \log K=6.1931-2963 / T+0.3 \log [P]$

Design Basis: May be the nominal (average), low or high nominal, or identical to safety basis as appropriate for the specific design case.

NA $=$ Not Applicable NDA = No Data Available at this time

\begin{tabular}{|c|l|l|}
\hline Endinate Ho. & DESIGiN BASIS VALUE & \multicolumn{1}{c|}{ Endinote No. } \\
\hline 46 & $\begin{array}{l}\text { The Design Basis } \\
\text { is the same as } \\
\text { the Safety Basis. }\end{array}$ & $\begin{array}{l}\text { See Safety } \\
\text { Basis } \\
\text { Endnote }\end{array}$ \\
42 & \\
6 & \\
46 & & \\
7 & & \\
7 & & \\
7 & & \\
7 & & \\
\end{tabular}

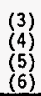


Table 4-3. KE \& KW Fuel Reaction Rate with Air Continued

KE \& KW FUEL REACTION RATE (with air as a function of temperature, pressure, \& humidity) Continued

Basic Rate Equation Rate $\left[\pi g(\right.$ th gain $) / \mathrm{hrl}=\hat{A}_{j} \xi \mathrm{K}$

$A_{a}=$ Gecometric surface Area of Uranium, $\mathrm{cm}^{2}$

$\xi^{3}=$ React ivity Enhancement Factor, Dimensionless
$K=$ Rate Constant (can be dependent on temperature and resctant concentration), mg(wt gain)/hr-c $\mathrm{cm}^{2}$

\begin{tabular}{|c|c|c|c|c|}
\hline & SAFETY BASIS VALUE & Endnote No. & DESIGN BASIS VALUE & Endnate No. \\
\hline $\begin{array}{l}\text { Rate } \\
\text { Constant } \\
\text { (K) }\end{array}$ & 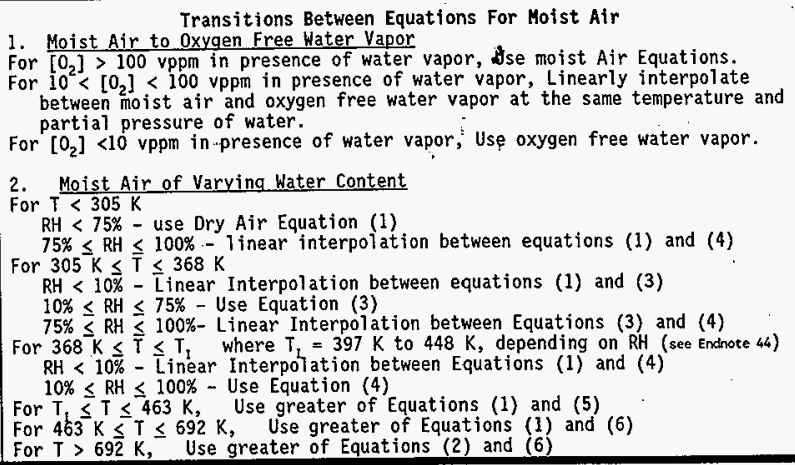 & 44 & $\begin{array}{l}\text { The Design Basis } \\
\text { is the same as } \\
\text { the Safety Basis. }\end{array}$ & $\begin{array}{l}\text { See Safety } \\
\text { Basis } \\
\text { Endnote }\end{array}$ \\
\hline
\end{tabular}

Definitions: NA $=$ Not Applicable NDA = No Data Available at this time 
Table 4-4. KE \& KW Reaction Rate with Water

KE \& KW FUEL REACTION RATE (with water as a function of temperature \& dissolved oxygen content)

Basic Rate Equation: Rate [mg(wt gain)/hr] $=A_{s} \xi K$

where: $\quad A_{s}=$ Geometric Surface Area of Uranium, $\mathrm{cm}^{2}$

$\xi=$ Reactivity Enhancement Factor, Dimensionless

$K=$ Rate Constant (can be dependent on temperature and reactant concentration), mg(wt gain)/hr-cm²

\begin{tabular}{|c|c|c|c|c|}
\hline & SAFETY BASIS VALUE & Endhote No. & DESIGN BASIS VALUE & Endinote Ho. \\
\hline $\begin{array}{l}\text { Rate } \\
\text { Constant } \\
\text { (K) }\end{array}$ & 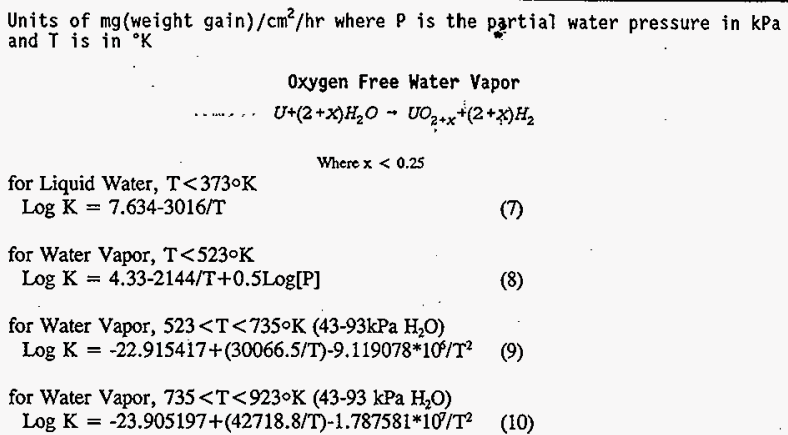 & $\begin{array}{l}45 \\
8\end{array}$ & $\begin{array}{l}\text { The Design Basis is } \\
\text { the same as the } \\
\text { Safety Basis. }\end{array}$ & $\begin{array}{l}\text { See Safety } \\
\text { Basis Endnote }\end{array}$ \\
\hline
\end{tabular}

Definitions:

Design Basis: May be the nominal (average), Jow or high nominal, or identical to safety basis as appropriate for the specific design case. NA = Not Appticable NDA = No Data Available at this time 
Table 4-5. Deleted

\section{Table 4-6. Radiolytic G Value}

RADIOLYTIC G VALUE

\begin{tabular}{|c|c|c|c|c|}
\hline. & SAFETY BASIS VALIEE & Endnote No. & DESIGN BASIS VALUE & Endnote No. \\
\hline $\begin{array}{l}\text { Oxygen Production } G \text { values as a } \\
\text { function of alpha radiation }\end{array}$ & $\begin{array}{l}0.75\left(X_{W}\right) \text { molecules } 0_{2} / 100 \text { eV absorbed by } \\
\text { particulate } 0 \leq X_{W} \leq 1 \\
\text { where: } \\
X_{w}=\text { Weight fraction water in fuel } \\
\text { particulate }\end{array}$ & 10 & $\begin{array}{l}\text { Oxygen generation is only a cansideration of the } \\
\text { safety basis, therefore the desisn basts is the } \\
\text { sarne as the safety basis. }\end{array}$ & $\begin{array}{l}\text { Same os Safety } \\
\text { Basis Endrote }\end{array}$ \\
\hline $\begin{array}{l}\text { Oxygen Production } G \text { values as a } \\
\text { function of beta radiation }\end{array}$ & $\begin{array}{l}0.225\left(X_{w}\right) \text { molecules } 0,100 \mathrm{eV} \text { absorbed } \\
\text { by particulate } 0 \leq X_{w} \leq 1 \text {. } \\
\text { where: } \\
X_{w}=\text { Weight fraction water in fuel } \\
\text { particulate }\end{array}$ & 11 & $\begin{array}{l}\text { oxygen generation is anly a consideration of the } \\
\text { safety basis, therefore the design basis is the } \\
\text { same as the safety basis. }\end{array}$ & $\begin{array}{l}\text { Same as Safety } \\
\text { Basis Endnote }\end{array}$ \\
\hline $\begin{array}{l}\text { Oxygen Production } G \text { values as a } \\
\text { function of gamma radiation }\end{array}$ & $\begin{array}{l}1\left(X_{w}\right) \text { molecules } 0_{2} / 100 \mathrm{eV} \text { absorbed by } \\
\text { particulate } 0 \leq X_{4} \leq 0.225 \\
0.225 \text { molecules } 0_{2} / 100 \mathrm{eV} \text { absorbed by } \\
\text { particulate } 0.225<X_{\mathrm{w}} \leq 1 \\
\text { where: } \\
X_{\mathrm{W}}=\text { Weight fraction water in fuel } \\
\text { particulate }\end{array}$ & 12 & $\begin{array}{l}\text { oxygen generation is only a consideration of the } \\
\text { safety basis, therefore the design basis is the } \\
\text { same os the safety basis. }\end{array}$ & $\begin{array}{l}\text { Same as Ssfety } \\
\text { Basis Endnote }\end{array}$ \\
\hline
\end{tabular}


Table 4-7. Canister Particulate Mass \& Water Content

CANISTER PARTICULATE MASS \& WATER CONTENT

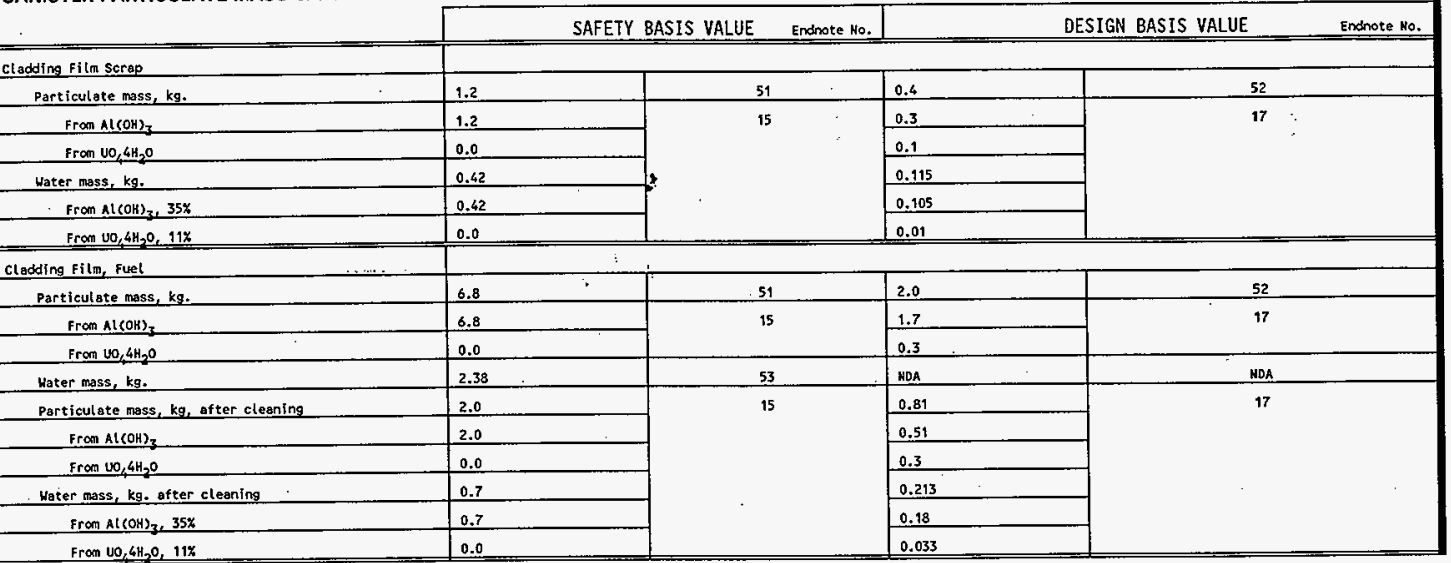

Definitions:

Design Basis: May be the nominal (average), low or high nominal, or identical to safety basis as appropriate for the specific design case. $N A=$ Not Applicable NDA = No Data Available at this time 
Table 4-7. Canister Particulate Mass \& Water Content

CANISTER PARTICULATE MASS \& WATER CONTENT

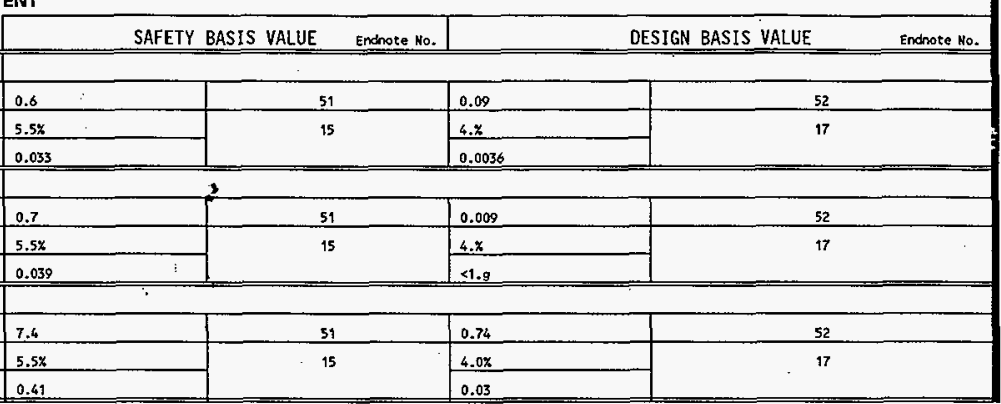

Definitions:

Design Basis: May be the nominal (average), low or high nominal, or identical to safety basis as appropriate for the specific design case. NA $=$ Not Applicable NDA = No Data Available at this time 
Table 4-7. Canister Particulate Mass \& Water Content

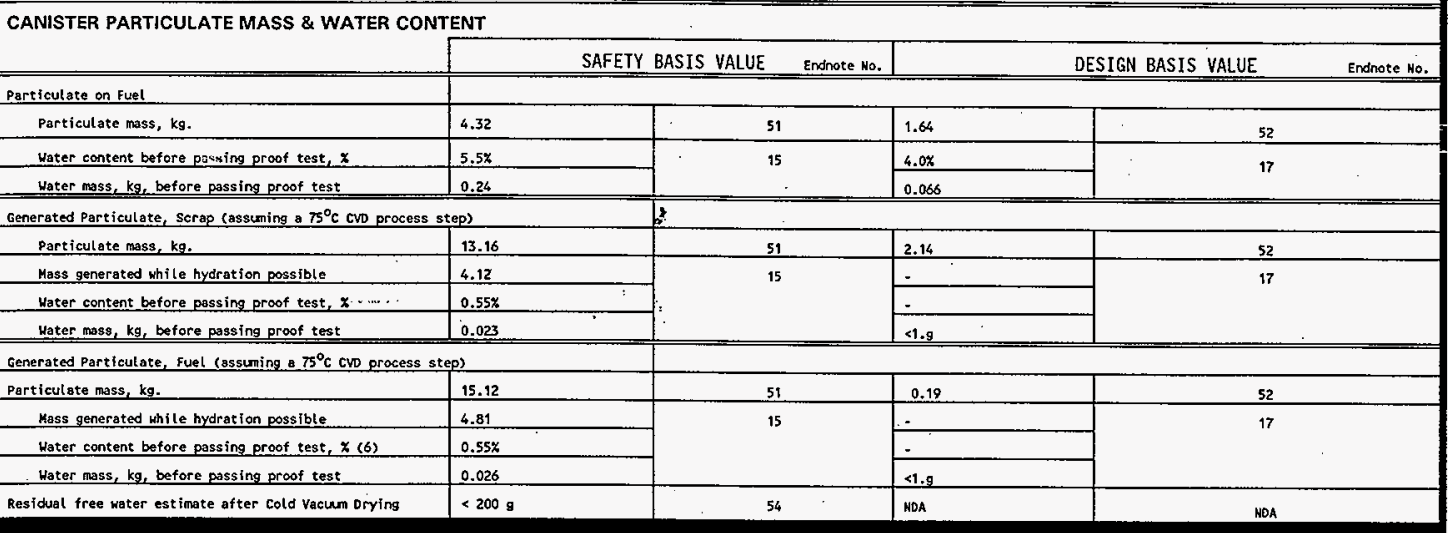

Table 4-7a. Deleted, See Section 5.0, Table 5-1.

Table 4-8. Deleted

Definitions:

Design Basis: May be the nominal (average), low or high nominal, or identical to safety basis as appropriate for the specific design case. NA = Not Applicable NDA = No Data Available at this time 
Table 4-9. Fuel Damage

\begin{tabular}{|c|c|c|c|c|c|c|c|}
\hline \multirow{2}{*}{\multicolumn{2}{|c|}{ FUEL DAMAGE }} & \multicolumn{3}{|c|}{ SAFETY BASIS VALUE } & \multicolumn{3}{|c|}{ DESIGN BASIS VALUE } \\
\hline & & Imer & outer & Endrote No. & Inner & outer & Endnote No. \\
\hline \multirow{4}{*}{ KE } & Intact Fuel - No evidence of cladding breach or deposited sludge. & $86 \%$ & $49 \%$ & \multirow[t]{4}{*}{19} & $86 \%$ & $49 \%$ & \multirow[t]{4}{*}{19} \\
\hline & $\begin{array}{l}\text { Breached Fuel - Minor cladding rupture with no reacted fue] or } \\
\text { deposited sludge visibly present. }\end{array}$ & $9 \%$ & $9 \%$ & & $9 \%$ & $9 \%$ & \\
\hline & $\begin{array}{l}\text { Defected Fuel - Definite evidence of cladding breach with reacted } \\
\text { fuel egressing from the element. The amount of exposed fuel may be } \\
\text { significant (e.g. loose end cap), but there is no gross } 2 \text { ladding } \\
\text { splitting, element dilation, or fuel voiding. }\end{array}$ & $4 \%$ & $38 \%$ & & $4 \%$ & $38 \%$ & \\
\hline & $\begin{array}{l}\text { Bad Fue1 - Gross failure is evident with substantial element } \\
\text { dilation, cladding splitting, fuel "mushrooming, " or: fuel voiding. }\end{array}$ & $1 \%$ & $4 \%$ & & $1 \%$ & $4 \%$ & \\
\hline & & \multicolumn{3}{|c|}{ SAFETY BASIS VALUE } & \multicolumn{3}{|c|}{ DESIGN BASIS VALUE } \\
\hline & & Inner & outer & Endrote No. & Inner & Outer & Endrote No. \\
\hline \multirow{4}{*}{$\dot{K} W$} & Intact Fuel - No evidence of cladding breach or deposited sludge. & $84 \%$ & $50 \%$ & \multirow[t]{4}{*}{19} & $84 \%$ & $50 \%$. & \multirow[t]{4}{*}{19} \\
\hline & $\begin{array}{l}\text { Breached Fuel - Minor cladding rupture with no reacted fuel or } \\
\text { deposited sludge visibly present. }\end{array}$ & $14 \%$ & $39 \%$ & & $14 \%$ & $39 \%$ & \\
\hline & $\begin{array}{l}\text { Defected Fuel - Definite evidence of cladding breach with reacted } \\
\text { fuel egressing from the element, but there is no gross cladding } \\
\text { spititting, element dilation, or fuel voiding. }\end{array}$ & $0 \%$ & $0 \%$ & & $0 \%$ & $0 \%$ & \\
\hline & $\begin{array}{l}\text { Bad Fuel - Gross failure is evident with substantial element } \\
\text { dilation, cladding splitting, fuel "mushrooming, or fuel voiding. }\end{array}$ & $2 \%$ & $11 \%$ & & $2 \%$ & $11 \%$ & \\
\hline
\end{tabular}

Definitions: NA $=$ Not Applicable NDA = No Data Available at this time 
Tạble 4-10. Deleted

Table 4-11. Fuel Decay / Heat Generation per MCO

FUEL DECAY / HEAT GENERATION per MCO

\begin{tabular}{|c|c|c|c|c|c|}
\hline & & SAFETY BASIS VALUE & ote No. & DESIGN BASIS VALUE & Endnote No. \\
\hline \multirow[t]{3}{*}{$\begin{array}{l}\text { FUEL } \\
\text { TYPES }\end{array}$} & Hark IV & $\begin{array}{l}776 \text { Watts Maximum } \\
\text { Heat generation in W/MTU (decayed to } 5 / 31 / 98 \text { ) } \\
=1420 \mathrm{~W}+11.6 \mathrm{MTU}=122.4 \mathrm{~W} / \mathrm{MTU} \\
270 \text { assembly/MCO } \times 23.48 \mathrm{kgU} / \text { assenbly }=6339.6 \mathrm{KgU} / \mathrm{MCO} \\
122.4 \mathrm{~W} / \mathrm{MTU} \times 6339.6 \mathrm{KgU} / \mathrm{MCO}=775.96 \mathrm{~W} / \mathrm{MCO} \\
\text { MOTE: } 5 \text { tfer baskets per MCO represents bounding ease. }\end{array}$ & $\begin{array}{l}21 \\
22\end{array}$ & \multirow{2}{*}{$\begin{array}{l}403 \text { Watts Average } \\
\text { (includes MarkIV \& Mark IA) } \\
\text { Total fuel heat generation in the combined basins } \\
=1.61 \text { e+05 } \mathrm{W} \text { fuel decayed to } 5 / 31 / 98 \\
161,000 \mathrm{~W} \text { total Heat load in KE+KW Basins }+ \\
400 \mathrm{MCO} s \text { (nominal to be processed }=403 \mathrm{~W} / \mathrm{MCO}\end{array}$} & \multirow{2}{*}{ ns } \\
\hline & $\begin{array}{l}\text { Mark IA } \\
\text { Mark tA Note: KE } \\
\text { Basin does not } \\
\text { conta in Mark IA } \\
\text { Fuel. }\end{array}$ & $\begin{array}{l}585 \text { Watts Maximum } \\
\text { Heat generation in W/MTU (decayed to } 5 / 31 / 98 \text { ) } \\
=1420 \mathrm{~W}+11.6 \mathrm{MTU}=122.4 \mathrm{~W} / \mathrm{MTU} \\
288 \text { assembly/HCO } \times 16.59 \mathrm{KgU} / \mathrm{assembly}=4777.92 \mathrm{KgU} / \mathrm{MCO} \\
122.4 \mathrm{~W} / \mathrm{MTU} \times 4777.92 \mathrm{KgU} / \mathrm{MCO}=585 \mathrm{~W} / \mathrm{MCO} \\
\text { HOrE: } 6 \text { tier baskets per } \mathrm{MCO} \text { represents bounding case. }\end{array}$ & $\begin{array}{l}21 \\
23\end{array}$ & & \\
\hline & $\begin{array}{l}\mathrm{SPR} \\
\text { SPR Yote: } \\
\mathrm{KE}+\mathrm{KH} S \mathrm{SPR}=3.3 \\
\mathrm{HTU} \text { rotal }\end{array}$ & $\begin{array}{l}329 \text { Watts } \\
\text { Total activity of selected radionuclides }=7.05 \mathrm{e}+04 \mathrm{Ci} \\
\text { (decayed to } 1 / 1 / 94 \text { ) } \\
\text { NoTE: All ser fuel will be contained in } 1 \text { Noc. }\end{array}$ & 27 & Same as Safety Basis & $\begin{array}{l}\text { See Safety 8asis } \\
\text { Endinote No. }\end{array}$ \\
\hline
\end{tabular}

Definitions:

Design Basis: May be the nominal (average), low or high nominal, or identical to safety basis as appropriate for the specific design case. $N A=$ Not Applicable

NDA = No Data Available at this time 
Table 4-12. N Fuel Source Terms

N FUEL SOURCE TERMS

Document WHC-SD-SNF-ANAL-011, titled "Comparison of Spent Fuel Databases for K Basin" compares the Safeguards Control and Accountability Transaction System (SCATS) and reconstructed SCATS (R-SCATS) databases. SCATS was the basis for both the COLDMAC database and the R-SCATS database. (See Endnote 28)

DATABASES used for the following purposes: SAFETY BASIS VALUE Endnote No. DESIGN BASIS VALUE Endnote No.

\begin{tabular}{|c|c|c|c|c|}
\hline $\begin{array}{l}\text { X Basin Fuel } \\
\text { Aecountability Location }\end{array}$ & NA & NA & $\begin{array}{l}\text { COLDMAC FUEL DATABASE is used } \\
\text { to produce WHC-IP-0069, "N/K } \\
\text { Fuels Inventory" }\end{array}$ & NA \\
\hline $\begin{array}{l}\text { K Basin SAR } \\
\text { safety/Regulatory } \\
\text { Assessment Basis }\end{array}$ & $\begin{array}{l}\text { R-SCATS FUEL DATABASE is used as the basis } \\
\text { for WHC-SD-NR-ANAL-014, "Conso? idated Fuel } \\
\text { Decay Heat Calculations" }\end{array}$ & NA & NA & NA \\
\hline $\begin{array}{l}\text { CSB and CVD SAR } \\
\text { Safety/Regulatory } \\
\text { Assessment Basis }\end{array}$ & $\begin{array}{l}\text { SCATS FUEL DATABASE is used as the basis for } \\
\text { WHC-SD-SNF-TI-009, "105 K Basin Material } \\
\text { Design Feed Description for SNF Project } \\
\text { Facilities" }\end{array}$ & NA & MA & NA \\
\hline \multirow[t]{2}{*}{$\begin{array}{l}\text { Shielding (CSB \& } \\
\text { CVD) }\end{array}$} & $\begin{array}{l}\text { Fission \& Activation } \\
\text { Product Totals } 4.05 \times 10^{6} \mathrm{ci} / \mathrm{MTU} \\
\text { Actinide Totals } \frac{1.01 \times 10^{4}}{5.06 \times 10^{4}} \mathrm{ci} / \mathrm{MTU}\end{array}$ & 29 & $2.61 \times 10^{4} \mathrm{ci} / \mathrm{MTU} * *$ & 41 \\
\hline & \multicolumn{2}{|l|}{ " MkIV fuel with $16 x$ Pu-240 aged 13.5 years, decayed to $5 / 31 / 98$} & \multicolumn{2}{|c|}{ 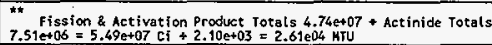 } \\
\hline
\end{tabular}

Definitions:

Design Basis: May be the nominal (average), low or high nominal, or identical to safety basis as appropriate for the specific design case. $\mathrm{NA}=$ Not Applicable NDA = No Data Avaifiable at this time 


\section{Table 4-12. N Fuel Source Terms Continued}

Radionuclide Inventory $K$ East Basin

...

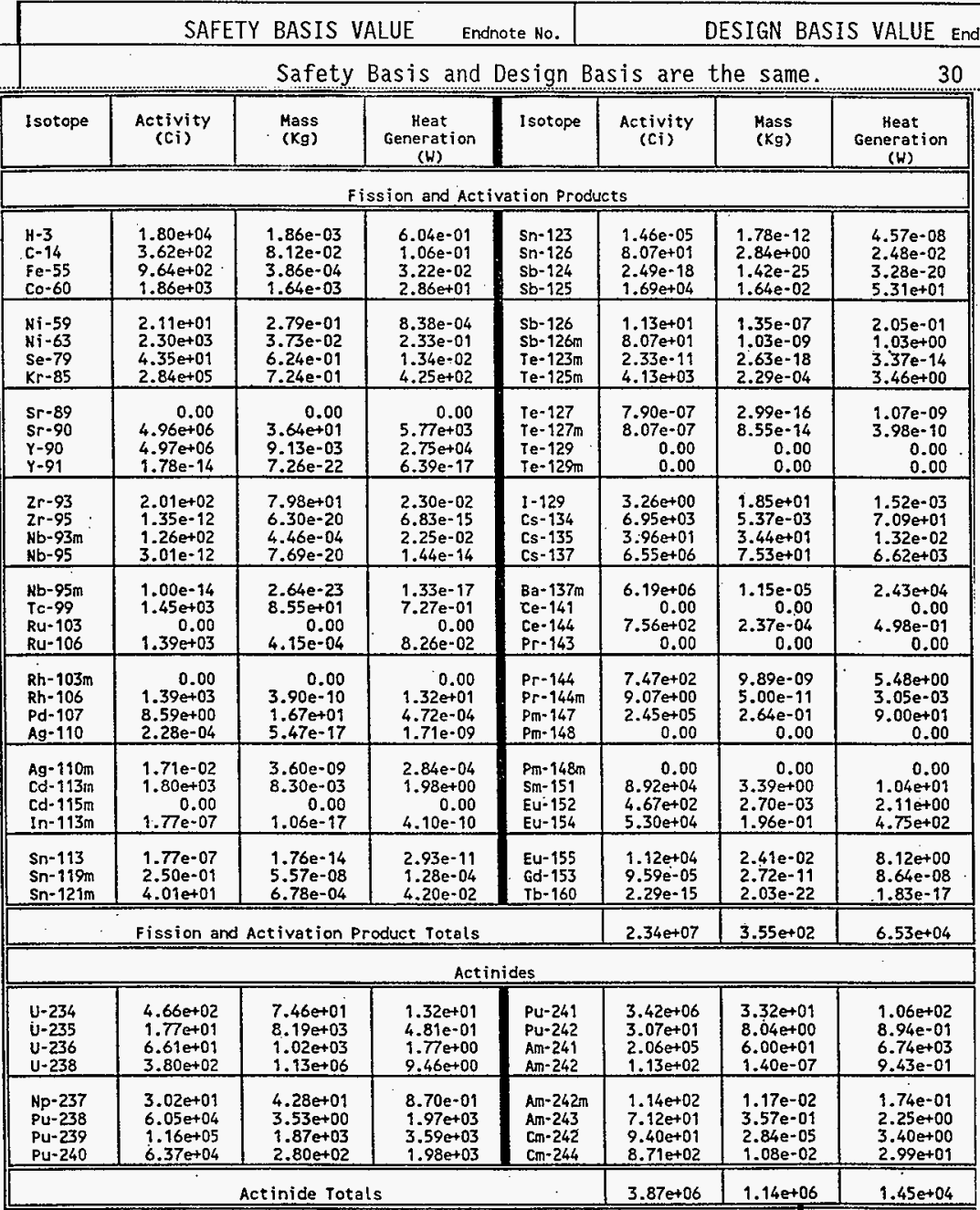

06/02/1997 RADNUC2A run for K-East Basin fuel. Results decayed to 05/31/1998. Total fuel mass in $\mathrm{X}$-East $1.15 \mathrm{e}+03$ MTU. Total fuel activity in $\mathrm{K}$-East $2.73 \mathrm{e}+0 \mathrm{C} \mathrm{C}$. Total fuel heat generation in $\mathrm{X}$-East $8.06 \mathrm{e}+04 \mathrm{H}$. Pu-239 and Pu-240 have been adjusted to ensure consistency with data previously reported by safeguards. 


\section{Table 4-12. N Fuel Source Terms Continued}

Radionuclide Inventiony $K$ West Basin

\begin{tabular}{|r|r|}
\hline SAFETY BASIS VALUE Endnote No. & DESIGN BASIS VALUE Endnote no. \\
\hline Safety Basis and Design Basis are the same.
\end{tabular}

\begin{tabular}{|c|c|c|c|c|c|c|c|}
\hline Isotope & $\begin{array}{l}\text { Activity } \\
\text { (Ci) }\end{array}$ & $\begin{array}{l}\text { Mass } \\
\text { (Kg) }\end{array}$ & $\begin{array}{c}\text { Heat } \\
\text { Generation } \\
\text { (W) }\end{array}$ & I sotope & $\begin{array}{l}\text { Activity } \\
\text { (ci) }\end{array}$ & $\begin{array}{l}\text { Mass } \\
(\mathrm{Kg})\end{array}$ & $\begin{array}{c}\text { Heat } \\
\text { Generation } \\
\text { (W) }\end{array}$ \\
\hline
\end{tabular}

\begin{tabular}{|c|c|c|c|c|c|c|c|}
\hline $\begin{array}{l}h-3 \\
C-14 \\
F e=55 \\
\text { Co- } 60\end{array}$ & $\begin{array}{l}1.86 e+04 \\
3.31 e+02 \\
8.75 e+02 \\
2.10 e+03 \\
\end{array}$ & $\begin{array}{l}1.93 e-03 \\
7.43 e-02 \\
3.50 e-04 \\
1.86 e-03 \\
\end{array}$ & $\begin{array}{l}6.24 e-01 \\
9.67 e-02 \\
2.92 e-02 \\
3.22 e+01 \\
\end{array}$ & $\begin{array}{l}\mathrm{Sn}-123 \\
\mathrm{Sn}-126 \\
\mathrm{sb}-124 \\
\mathrm{sb}-125\end{array}$ & $\begin{array}{l}2.75 \mathrm{e}-06 \\
7.50 \mathrm{e}+01 \\
5.45 \mathrm{e}-19 \\
1.66 \mathrm{e}+04\end{array}$ & $\begin{array}{l}3.35 e-13 \\
2.64 \mathrm{e}+00 \\
3.12 \mathrm{e}-26 \\
1.61 \mathrm{e}-02 \\
\end{array}$ & $\begin{array}{l}8.58 \mathrm{e}-09 \\
2.30 \mathrm{e}-02 \\
7.21 \mathrm{e}-21 \\
5.23 \mathrm{e}+01\end{array}$ \\
\hline $\begin{array}{l}\mathrm{Ni}-59 \\
\mathrm{Ni}-63 \\
\mathrm{Se}-79 \\
\mathrm{Kr}-85\end{array}$ & $\begin{array}{l}1.99 \mathrm{e}+01 \\
2.19 \mathrm{e}+03 \\
4.28 \mathrm{e}+01 \\
3.06 \mathrm{e}+05 \\
\end{array}$ & $\begin{array}{l}2.63 e-01 \\
3.55 e-02 \\
6.14 e-01 \\
7.80 e-01 \\
\end{array}$ & $\begin{array}{l}7.88 \mathrm{e}-04 \\
2.21 \mathrm{e}-01 \\
1.32 \mathrm{e}-02 \\
4.60 \mathrm{e}+02 \\
\end{array}$ & $\begin{array}{l}S b=126 \\
S b-126 m \\
T e-123 m \\
T e-125 m\end{array}$ & $\begin{array}{l}1.05 \mathrm{e}+01 \\
7.50 \mathrm{e}+01 \\
4.28 \mathrm{e}-12 \\
4.05 \mathrm{e}+03\end{array}$ & $\begin{array}{l}1.26 e-07 \\
9.55 e-10 \\
4.82 e-19 \\
2.25 e-04 \\
\end{array}$ & $\begin{array}{l}1.90 \mathrm{e}-01 \\
9.61 \mathrm{e}-01 \\
6.21 \mathrm{e}-15 \\
3.40 \mathrm{e}+00 \\
\end{array}$ \\
\hline $\begin{array}{l}\$ r-89 \\
\$ r-90 \\
Y-90 \\
Y-91 \\
\end{array}$ & $\begin{array}{r}0.00 \\
5.17 \mathrm{e}+06 \\
5.17 \mathrm{e}+06 \\
4.47 \mathrm{e}-15 \\
\end{array}$ & $\begin{array}{r}0.00 \\
3.79 \mathrm{e}+01 \\
9.50 \mathrm{e}-03 \\
1.82 \mathrm{e}-22 \\
\end{array}$ & $\begin{array}{r}0.00 \\
6.01 \mathrm{e}+03 \\
2.86 \mathrm{e}+04 \\
1.60 \mathrm{e}-17 \\
\end{array}$ & $\begin{array}{l}\text { Te-127 } \\
\text { Te-127m } \\
T e-129 \\
T e-129 \mathrm{~m}\end{array}$ & $\begin{array}{r}1.59 \mathrm{e}-07 \\
1.62 \mathrm{e}-07 \\
0.00 \\
0.00 \\
\end{array}$ & $\begin{array}{r}6.02 e-17 \\
1.72 e-14 \\
0.00 \\
0.00 \\
\end{array}$ & $\begin{array}{r}2.14 \mathrm{e}-10 \\
8.03 \mathrm{e}-11 \\
0.00 \\
.0 .00 \\
\end{array}$ \\
\hline $\begin{array}{l}2 r-93 \\
2 r-95 \\
N b-93 m \\
N b-95\end{array}$ & $\begin{array}{l}2.01 \mathrm{e}+02 \\
3.30 \mathrm{e}-13 \\
1.22 \mathrm{e}+02 \\
7.33 \mathrm{e}-13\end{array}$ & $\begin{array}{l}7.98 \mathrm{e}+01 \\
1.54 \mathrm{e}-20 \\
4.32 \mathrm{e}-04 \\
1.87 \mathrm{e}-20 \\
\end{array}$ & $\begin{array}{l}2.30 e-02 \\
1.66 e-15 \\
2.18 e-02 \\
3.52 e-15\end{array}$ & $\begin{array}{l}t-129 \\
C s-134 \\
C s-135 \\
C s-137\end{array}$ & $\begin{array}{l}3.11 \mathrm{e}+00 \\
8.96 \mathrm{e}+03 \\
3.79 \mathrm{e}+01 \\
6.64 \mathrm{e}+06\end{array}$ & $\begin{array}{l}1.76 \mathrm{e}+01 \\
6.92 \mathrm{e}-03 \\
3.29 \mathrm{e}+01 \\
7.63 \mathrm{e}+01\end{array}$ & $\begin{array}{l}1.46 \mathrm{e}-03 \\
9.14 \mathrm{e}+01 \\
1.27 \mathrm{e}-02 \\
6.71 \mathrm{e}+03\end{array}$ \\
\hline $\begin{array}{l}\text { Nb- } 95 \mathrm{~m} \\
\text { Tc- } 99 \\
\text { Ru- } 103 \\
\text { Ru- } 106 \\
\end{array}$ & $\begin{array}{r}2.45 \mathrm{e}-15 \\
1.43 \mathrm{e}+03 \\
0.00 \\
4.34 \mathrm{e}+02 \\
\end{array}$ & $\begin{array}{r}6.44 \mathrm{e}-24 \\
8.43 \mathrm{e}+01 \\
0.00 \\
1.30 \mathrm{e}-04 \\
\end{array}$ & $\begin{array}{r}3.23 \mathrm{e}-18 \\
7.18 \mathrm{e}-01 \\
0.00 \\
2.58 \mathrm{e}-02 \\
\end{array}$ & $\begin{array}{l}\mathrm{Ba}-137 \mathrm{~m} \\
\mathrm{Ce}-141 \\
\mathrm{Ce}-144 \\
\mathrm{Pr}-143 \\
\end{array}$ & $\begin{array}{r}6.28 \mathrm{e}+06 \\
0.00 \\
1.58 \mathrm{e}+02 \\
0.00 \\
\end{array}$ & $\begin{array}{r}1.17 e-05 \\
0.00 \\
4.95 e-05 \\
0.00 \\
\end{array}$ & $\begin{array}{r}2.46 \mathrm{e}+04 \\
0.00 \\
1.04 \mathrm{e}-01 \\
0.00 \\
\end{array}$ \\
\hline $\begin{array}{l}\mathrm{Rh}-103 \mathrm{~m} \\
\mathrm{Rh}=106 \\
\mathrm{Pd}=107 \\
\mathrm{Ag}=110 \\
\end{array}$ & $\begin{array}{r}0.00 \\
4.34 \mathrm{e}+02 \\
7.68 \mathrm{e}+00 \\
5.66 \mathrm{e}-05 \\
\end{array}$ & $\begin{array}{r}0.00 \\
1.22 \mathrm{e}-10 \\
1.49 \mathrm{e}+01 \\
1.36 \mathrm{e}-17 \\
\end{array}$ & $\begin{array}{r}0.00 \\
4.13 \mathrm{e}+00 \\
4.22 \mathrm{e}-04 \\
4.25 \mathrm{e}-10 \\
\end{array}$ & $\begin{array}{l}\mathrm{Pr}-144 \\
\mathrm{Pr}-144 \mathrm{~m} \\
\mathrm{Pm}-147 \\
\mathrm{Pm}-148\end{array}$ & $\begin{array}{r}1.56 \mathrm{e}+02 \\
1.90 \mathrm{e}+00 \\
2.17 \mathrm{e}+05 \\
0.00\end{array}$ & $\begin{array}{r}2.06 \mathrm{e}-09 \\
1.05 \mathrm{e}-11 \\
2.34 \mathrm{e}-01 \\
0.00\end{array}$ & $\begin{array}{r}1.15 \mathrm{e}+00 \\
6.39 \mathrm{e}-04 \\
7.97 \mathrm{e}+01 \\
0.00\end{array}$ \\
\hline $\begin{array}{l}\text { Ag-110m } \\
\text { Cd-113m } \\
\text { Cd-115m } \\
\text { In-113m }\end{array}$ & $\begin{array}{r}4.26 \mathrm{e}-03 \\
1.75 \mathrm{e}+03 \\
0.00 \\
3.74 \mathrm{e}-08 \\
\end{array}$ & $\begin{array}{r}8.97 \mathrm{e}-10 \\
8.07 \mathrm{e}-03 \\
0.00 \\
2.24 \mathrm{e}-18 \\
\end{array}$ & $\begin{array}{r}7.06 \mathrm{e}-05 \\
1.92 \mathrm{e}+00 \\
0.00 \\
8.64 \mathrm{e}-11 \\
\end{array}$ & $\begin{array}{l}P m-148 m \\
S m-151 \\
\text { Eu-152 } \\
\text { Eu-154 }\end{array}$ & $\begin{array}{r}0.00 \\
8.66 \mathrm{e}+04 \\
4.77 \mathrm{e}+02 \\
5.44 \mathrm{e}+04 \\
\end{array}$ & $\begin{array}{r}0.00 \\
3.29 \mathrm{e}+00 \\
2.76 \mathrm{e}-03 \\
2.02 \mathrm{e}-01 \\
\end{array}$ & $\begin{array}{r}0.00 \\
1.01 \mathrm{e}+01 \\
2.16 \mathrm{e}+00 \\
4.89 \mathrm{e}+02 \\
\end{array}$ \\
\hline $\begin{array}{l}S n-113 \\
s n-119 m \\
S n-121 m \\
\end{array}$ & $\begin{array}{l}3.74 e-08 \\
4.75 e-02 \\
3.95 e+01 \\
\end{array}$ & $\begin{array}{r}3.73 e-15 \\
1.06 \mathrm{e}-08 \\
6.68 \mathrm{e}-04 \\
\end{array}$ & $\begin{array}{l}6.21 e-12 \\
2.45 e-05 \\
4.15 e-02 \\
\end{array}$ & $\begin{array}{l}\text { Eu-155 } \\
G d-153 \\
T b-160 \\
\end{array}$ & $\begin{array}{l}1.08 e+04 \\
3.23 e-05 \\
4.77 e-16 \\
\end{array}$ & $\begin{array}{l}2.32 e-02 \\
9.16 e-12 \\
4.23 e-23\end{array}$ & $\begin{array}{l}7.85 e+00 \\
2.91 e-08 \\
3.81 e-18 \\
\end{array}$ \\
\hline \multicolumn{5}{|c|}{ Fission and Activation Product Totals } & $2.40 \mathrm{e}+07$ & $3.52 \mathrm{e}+02$ & $6.72 e+04$ \\
\hline \multicolumn{8}{|c|}{ Actinides } \\
\hline $\begin{array}{l}U-234 \\
U-235 \\
U-236 \\
U-238 \\
\end{array}$ & $\begin{array}{l}4.08 \mathrm{e}+02 \\
1.60 \mathrm{e}+01 \\
6.11 \mathrm{e}+01 \\
3.16 \mathrm{e}+02 \\
\end{array}$ & $\begin{array}{l}6.53 \mathrm{e}+01 \\
7.40 \mathrm{e}+03 \\
9.44 \mathrm{e}+02 \\
9.40 \mathrm{e}+05 \\
\end{array}$ & $\begin{array}{l}1.15 \mathrm{e}+01 \\
4.34 \mathrm{e}-01 \\
1.63 \mathrm{e}+00 \\
7.85 \mathrm{e}+00 \\
\end{array}$ & $\begin{array}{l}P u-241 \\
P u-242 \\
A m-241 \\
A m-242 \\
\end{array}$ & $\begin{array}{l}3.26 \mathrm{e}+06 \\
2.42 \mathrm{e}+01 \\
1.69 \mathrm{e}+05 \\
8.15 \mathrm{e}+01 \\
\end{array}$ & $\begin{array}{l}3.16 \mathrm{e}+01 \\
6.34 \mathrm{e}+00 \\
4.92 \mathrm{e}+01 \\
1.01 \mathrm{e}-07\end{array}$ & $\begin{array}{l}1.01 \mathrm{e}+02 \\
7.03 \mathrm{e}-01 \\
5.54 \mathrm{e}+03 \\
6.77 \mathrm{e}-01 \\
\end{array}$ \\
\hline $\begin{array}{l}N p-237 \\
P u-238 \\
P u-239 \\
P u-240 \\
\end{array}$ & $\begin{array}{l}2.70 \mathrm{e}+01 \\
5.10 \mathrm{e}+04 \\
1.01 \mathrm{e}+05 \\
5.53 \mathrm{e}+04 \\
\end{array}$ & $\begin{array}{l}3.83 \mathrm{e}+01 \\
2.98 \mathrm{e}+00 \\
1.63 \mathrm{e}+03 \\
2.42 \mathrm{e}+02 \\
\end{array}$ & $\begin{array}{l}7.79 \mathrm{e}-01 \\
1.66 \mathrm{e}+03 \\
3.13 \mathrm{e}+03 \\
1.72 \mathrm{e}+03 \\
\end{array}$ & $\begin{array}{l}\mathrm{Am}-242 \mathrm{n} \\
\mathrm{Am}-243 \\
\mathrm{Cm}-242 \\
\mathrm{Cm}-244\end{array}$ & $\begin{array}{l}8.19 \mathrm{e}+01 \\
4.89 \mathrm{e}+01 \\
6.76 \mathrm{e}+01 \\
5.72 \mathrm{e}+02 \\
\end{array}$ & $\begin{array}{l}8.43 e-03 \\
2.45 e-01 \\
2.04 e-05 \\
7.07 e-03 \\
\end{array}$ & $\begin{array}{l}1.25 e-01 \\
1.54 e+00 \\
2.45 e+00 \\
1.97 e+01 \\
\end{array}$ \\
\hline \multicolumn{5}{|c|}{ Actinide Totals } & $3.64 \mathrm{e}+06$ & $9.50 \mathrm{e}+05$ & $1.22 \mathrm{e}+04$ \\
\hline
\end{tabular}

06/02/4997 RADNUC2A run for K-Hest Basin. Results decayed to 05/31/1998. Total fuel mass in $K-$ West $9.53 \mathrm{e}+02$ MTU. Total fuel activity in K-West $2.77 \mathrm{e}+07 \mathrm{Ci}$. Total Heat Generation of fuel in K-Hest $8.03 e+04 W$. Pu-239 and Pu-240 have been adjusted to ensure consistency with data previously reported by
Safeguards. 
Table 4-12. N Fuel Source Terms Continued

Chemical Inventory for Both KE and KW Basins

SAFETY BASIS VALUE Endnote No. DESIGN BASIS VALUE Endnote No.

Safety Basis and Design Basis are the same.

\begin{tabular}{|c|c|c|c|c|}
\hline Element & $\begin{array}{c}\text { Uranium Alloy } 601 \\
(\mathrm{~kg})\end{array}$ & $\begin{array}{c}\text { zircaloy-2 cladding. } \\
(\mathrm{kg})\end{array}$ & $\begin{array}{c}\begin{array}{c}\text { Braze Filler } \\
(\mathrm{kg})\end{array} \\
\end{array}$ & $\begin{array}{l}\text { Totals } \\
(\mathrm{kg})^{2} \\
\end{array}$ \\
\hline $\begin{array}{c}\mathrm{Al} \\
B \\
\mathrm{Be} \\
\mathrm{C} \\
\end{array}$ & $\begin{array}{c}1,480 \div 1,900 \\
0.530^{\circ} \\
21.0 \\
769=1,550 \\
\end{array}$ & $\begin{array}{r}11.9 \\
0.074 \\
40.7 \\
\end{array}$ & $\begin{array}{c}0.411 \\
0.00142 \\
142 \\
1.42 \\
\end{array}$ & $\begin{array}{l}1.700 \\
0.605 \\
163 \\
1.200 \\
\end{array}$ \\
\hline $\begin{array}{l}\mathrm{Cd} \\
\mathrm{Co} \\
\mathrm{Cr} \\
\mathrm{Cu}\end{array}$ & $\begin{array}{r}0.530 \\
. \\
137 \\
158 \\
\end{array}$ & $\begin{array}{c}0.074 \\
1.48 \\
74-222 \\
7.40 \\
\end{array}$ & $\begin{array}{c}0.00142 \\
0.0567 \\
1.42 \cdot 4.26 \\
0.770 \\
\end{array}$ & $\begin{array}{l}0.605 \\
1.54 \\
288 \\
166 \\
\end{array}$ \\
\hline $\begin{array}{l}\mathrm{Fe} \\
\mathrm{H} \\
\mathrm{Hf} \\
\mathrm{Hg} \\
\end{array}$ & $\begin{array}{c}632-843 \\
4.22 \\
- \\
52.7 \\
\end{array}$ & $\begin{array}{c}104-296 \\
3.70 \\
29.6 \\
2.96 \\
\end{array}$ & $\begin{array}{c}1.70=5.96 \\
0.142 \\
0.567 \\
0.170 \\
\end{array}$ & $\begin{array}{l}941 \\
8.06 \\
30.2 \\
55.8 \\
\end{array}$ \\
\hline $\begin{array}{c}\mathrm{Mn} \\
\mathrm{Mo} \\
\mathrm{H} \\
\mathrm{Ha}\end{array}$ & $\begin{array}{c}52.7 \\
-\quad 158 \\
- \\
\end{array}$ & $\begin{array}{r}7.40 \\
7.40 \\
11.8 \\
2.96 \\
\end{array}$ & $\begin{array}{l}0.170 \\
0.142 \\
0.567 \\
0.0567\end{array}$ & $\begin{array}{l}60.3 \\
7.54 \\
170 \\
3.02 \\
\end{array}$ \\
\hline $\begin{array}{c}\mathrm{Mi} \\
0 \\
\mathrm{~Pb} \\
\mathrm{Si} \\
\end{array}$ & $\begin{array}{r}211 \\
\div \\
261 \\
\end{array}$ & $\begin{array}{c}44.4=118 \\
14.8 \\
14.8 \\
\end{array}$ & $\begin{array}{c}0.851-2.27 \\
6.53 \\
0.369 \\
0.709 \\
\end{array}$ & $\begin{array}{r}294 \\
6.53 \\
15.2 \\
-\quad 277 \\
\end{array}$ \\
\hline $\begin{array}{r}S n \\
Z i \\
V \\
H \\
2 r \\
\end{array}$ & $\begin{array}{c}- \\
\vdots \\
753 \\
\end{array}$ & $\begin{array}{c}1,780-2,520 \\
7.40 \\
7.40 \\
7.40 \\
145,000 \\
\end{array}$ & $\begin{array}{c}32.3-48.2 \\
0.142 \\
0.142 \\
0.284 \\
2.780 \\
\end{array}$ & $\begin{array}{r}2,190 \\
7.54 \\
7.54 \\
7.68 \\
148,000 \\
\end{array}$ \\
\hline \multicolumn{5}{|l|}{ Actinides } \\
\hline $\begin{array}{l}U \\
\mathrm{Mp} \\
\mathrm{Pu} \\
\mathrm{Am} \\
\mathrm{Cm} \\
\end{array}$ & $\begin{array}{c}2,100,000 \\
81.1 \\
4,120 \\
109 \\
0.018\end{array}$ & $\begin{array}{c}0.518 \\
\vdots \\
\vdots\end{array}$ & $\begin{array}{c}0.0113 \\
\vdots \\
\vdots\end{array}$ & $\begin{array}{c}2,100,000 \\
81.1 \\
4,120 \\
109 \\
0.018\end{array}$ \\
\hline \multicolumn{5}{|c|}{ Fission Products $2 \mathrm{a}$} \\
\hline $\begin{array}{l}\mathrm{Se} \\
\mathrm{Sr} \\
\mathrm{TC} \\
\mathrm{Pd} \\
\mathrm{Kr}\end{array}$ & $\begin{array}{r}12.0 \\
152 \\
170 \\
\quad \quad 133 \\
\quad 7.1 \\
\end{array}$ & $\vdots$ & $\begin{array}{r}\cdot \\
\vdots \\
\end{array}$ & $\begin{array}{r}12.0 \\
152 \\
170 \\
733 \\
77.1 \\
\end{array}$ \\
\hline $\begin{array}{l}1 \\
\mathrm{cs} . \\
\mathrm{Pm} \\
\mathrm{sin} \\
\mathrm{xe} \\
\end{array}$ & $\begin{array}{r}46.1 \\
4.65 \\
0.50 \\
176 \\
1040 \\
\end{array}$ & : & : & $\begin{array}{r}46.1 \\
4.65 \\
0.50 \\
176 \\
1040 \\
\end{array}$ \\
\hline
\end{tabular}

For the values with a range, the midpoint of the range is used.

stable fission product are based on the ratio of ${ }^{99} \mathrm{Tc}$ in Table 3.5 and the relative concentration of stable fission products from ORIGEH2 for $12 \% 240$ PU Mark IV assertities. 


\section{Table 4-12. N Fuel Source Terms Continued}

Shielding Design Basis

Based on Mk IV fuel at $16 \% \mathrm{Pu}-240$ aged 13.5 years.

\begin{tabular}{|r|r|r|}
\hline SAFETY BASIS VALUE Endnote No. & DESIGN BASIS VALUE Endnote No. \\
\hline
\end{tabular}

Safety Basis and Design Basis are the same. 33

\begin{tabular}{|c|c|c|c|c|c|}
\hline Isotope & $\begin{array}{l}\text { Activity } \\
\text { (Ci/MTU) }\end{array}$ & $\begin{array}{c}\text { Heat } \\
\text { Generation } \\
\text { (H/MTU) }\end{array}$ & I sotope & $\begin{array}{l}\text { Activity } \\
\text { (Ci/MTU) }\end{array}$ & $\begin{array}{c}\text { Heat } \\
\text { Generation } \\
\text { (W/MTU) }\end{array}$ \\
\hline \multicolumn{6}{|c|}{ Fission and Activation Products } \\
\hline $\begin{array}{l}H-3 \\
\mathrm{C}-14 \\
\mathrm{Fe}-55 \\
\mathrm{CO}-60 \\
\end{array}$ & $\begin{array}{l}4.03 \mathrm{e}+01 \\
5.27 \mathrm{e}-01 \\
5.23 \mathrm{e}+00 \\
6.27 \mathrm{e}+00 \\
\end{array}$ & $\begin{array}{l}1.36 e-03 \\
1.54 e-04 \\
1.75 e-04 \\
9.64 e-02\end{array}$ & $\begin{array}{l}S n-123 \\
S n=126 \\
S b-124 \\
s b-125 \\
\end{array}$ & $\begin{array}{r}4.08 \mathrm{e}-09 \\
1.22 \mathrm{e}-01 \\
0.00 \\
0.00 \\
\end{array}$ & $\begin{array}{r}1.26 \mathrm{e}-11 \\
3.75 \mathrm{e}-05 \\
0.00 \\
0.00 \\
\end{array}$ \\
\hline $\begin{array}{l}\text { Ni }-59 \\
\mathrm{Ni}-63 \\
\mathrm{Se}-79 \\
\mathrm{Kr} \cdot 85 \\
\end{array}$ & $\begin{array}{l}3.03 \mathrm{e}-02 \\
3.53 \mathrm{e}+00 \\
6.23 \mathrm{e}-02 \\
6.23 \mathrm{e}+02 \\
\end{array}$ & $\begin{array}{l}1.21 \mathrm{e}-06 \\
3.57 \mathrm{e}-04 \\
1.93 \mathrm{e}-05 \\
9.33 \mathrm{e}-01 \\
\end{array}$ & $\begin{array}{l}\text { Sb- } 126 \\
\text { sb- } 126 \mathrm{~m} \\
T e-123 \mathrm{~m} \\
T e-125 \mathrm{~m} \\
\end{array}$ & $\begin{array}{r}1.71 \mathrm{e}-02 \\
1.22 \mathrm{e}-01 \\
1.19 \mathrm{e}-13 \\
0.00 \\
\end{array}$ & $\begin{array}{r}3.10 e^{-04} \\
1.57 e^{-03} \\
1.72 e^{-16} \\
0.00 \\
\end{array}$ \\
\hline $\begin{array}{l}s r-89 \\
s r-90 \\
\gamma-90 \\
\gamma-91 \\
\end{array}$ & $\begin{array}{r}0.00 \\
8.19 \mathrm{e}+03 \\
8.19 \mathrm{e}+03 \\
0.00 \\
\end{array}$ & $\begin{array}{r}0.00 \\
9.50 \mathrm{e}+00 \\
4.53 \mathrm{e}+01 \\
0.00 \\
\end{array}$ & $\begin{array}{l}\text { Te-127 } \\
\text { Te-127m } \\
\text { Te-129 } \\
\text { Te-129m } \\
\end{array}$ & $\begin{array}{r}1.16 \mathrm{e}-10 \\
1.19 \mathrm{e}-10 \\
0.00 \\
0.00 \\
\end{array}$ & $\begin{array}{r}1.57 \mathrm{e}-13 \\
5.88 \mathrm{e}-14 \\
0.00 \\
0.00 \\
\end{array}$ \\
\hline $\begin{array}{l}2 r-93 \\
2 r-95 \\
\mathrm{Hb}-93 \mathrm{~m} \\
\mathrm{Nb}-95 \\
\end{array}$ & $\begin{array}{l}2.83 e-01 \\
4.20 \mathrm{e}-18 \\
1.38 \mathrm{e}-01 \\
9.33 \mathrm{e}-18 \\
\end{array}$ & $\begin{array}{l}3.25 \mathrm{e}-05 \\
2.12 \mathrm{e}-20 \\
2.47 \mathrm{e}-05 \\
4.48 \mathrm{e}-20 \\
\end{array}$ & $\begin{array}{l}I-129 \\
C s-134 \\
C s-135 \\
C s-137 \\
\end{array}$ & $\begin{array}{l}4.88 \mathrm{e}-03 \\
1.07 \mathrm{e}+02 \\
5.77 \mathrm{e}-02 \\
1.13 \mathrm{e}+04 \\
\end{array}$ & $\begin{array}{l}2.29 \mathrm{e}-06 \\
1.10 \mathrm{e}+00 \\
1.93 \mathrm{e}-05 \\
1.14 \mathrm{e}+01 \\
\end{array}$ \\
\hline $\begin{array}{l}\text { Nb- } 95 \mathrm{~m} \\
\mathrm{Tc}-99 \\
\text { Ru- } 103 \\
\text { Ru- } 106 \\
\end{array}$ & $\begin{array}{r}3.12 \mathrm{e}-20 \\
2.08 \mathrm{e}+00 \\
0.00 \\
9.38 \mathrm{e}+00 \\
\end{array}$ & $\begin{array}{r}4.12 \mathrm{e}-23 \\
1.05 \mathrm{e}-03 \\
0.00 \\
0.59 \mathrm{e}-04 \\
\end{array}$ & $\begin{array}{l}\text { Ba-137m } \\
\mathrm{Ce}-141 \\
\mathrm{Ce}-144 \\
\mathrm{Pr}=143 \\
\end{array}$ & $\begin{array}{r}1.07 \mathrm{e}+04 \\
0.00 \\
1.75 \mathrm{e}+00 \\
0.00 \\
\end{array}$ & $\begin{array}{r}4.18 \mathrm{e}+01 \\
0.00 \\
-1.15 \mathrm{e}-03 \\
0.00 \\
\end{array}$ \\
\hline $\begin{array}{l}\text { Rh-103m } \\
\text { Rh-106 } \\
\text { Pd-107 } \\
\text { Ag-110 } \\
\end{array}$ & $\begin{array}{r}0.00 \\
9.38 \mathrm{e}+00 \\
1.44 \mathrm{e}-02 \\
4.15 \mathrm{e}-06 \\
\end{array}$ & $\begin{array}{r}0.00 \\
8.97 \mathrm{e}-02 \\
7.94 \mathrm{e}-07 \\
3.11 \mathrm{e}-11 \\
\end{array}$ & $\begin{array}{l}\mathrm{Pr}-144 \\
\mathrm{Pr}-144 \mathrm{n} \\
\mathrm{Pm}-147 \\
\mathrm{Pm}-148 \\
\end{array}$ & $\begin{array}{r}1.73 \mathrm{e}+00 \\
2.10 \mathrm{e}-02 \\
1.06 \mathrm{e}+03 \\
0.00 \\
\end{array}$ & $\begin{array}{r}1.26 e-02 \\
7.05 e-06 \\
3.88 e-01 \\
0.00 \\
\end{array}$ \\
\hline $\begin{array}{l}\text { Ag- } 110 \mathrm{~m} \\
\mathrm{Cd}-113 \mathrm{~m} \\
\mathrm{Cd}-115 \mathrm{~m} \\
\mathrm{In}-113 \mathrm{~m} \\
\end{array}$ & $\begin{array}{r}3.12 \mathrm{e}-04 \\
3.96 \mathrm{e}+00 \\
0.00 \\
2.59 \mathrm{e}-11 \\
\end{array}$ & $\begin{array}{r}5.18 \mathrm{e}-06 \\
4.35 \mathrm{e}-03 \\
0.00 \\
5.98 \mathrm{e}-14 \\
\end{array}$ & $\begin{array}{l}\mathrm{Pm}-148 \mathrm{~m} \\
\mathrm{Sm}-151 \\
\mathrm{Eu}-152 \\
\mathrm{Eu}-154 \\
\end{array}$ & $\begin{array}{r}0.00 \\
1.08 e+02 \\
1.22 e+00 \\
2.02 e+02 \\
\end{array}$ & $\begin{array}{r}0.00 \\
1.26 \mathrm{e}-02 \\
5.50 \mathrm{e}-03 \\
1.81 \mathrm{e}+00 \\
\end{array}$ \\
\hline $\begin{array}{l}\mathrm{Sn}-113 \\
\mathrm{Sn}-119 \mathrm{~m} \\
\mathrm{sn}-121 \mathrm{~m} \\
\end{array}$ & $\begin{array}{r}2.59 \mathrm{e}-11 \\
0.00 \\
0.00 \\
\end{array}$ & $\begin{array}{r}4.30 \mathrm{e}-15 \\
0.00 \\
0.00 \\
\end{array}$ & $\begin{array}{l}\text { Eu-155 } \\
\text { Gd- } 153 \\
\text { Tb-160 } \\
\end{array}$ & $\begin{array}{l}3.42 \mathrm{e}+01 \\
3.92 \mathrm{e}-06 \\
2.22 \mathrm{e}-19 \\
\end{array}$ & $\begin{array}{l}2.48 \mathrm{e}-02 \\
3.53 \mathrm{e}-09 \\
1.78 \mathrm{e}-21 \\
\end{array}$ \\
\hline \multicolumn{4}{|c|}{ Fission and Activation Product Totals } & $4.05 \mathrm{e}+04$ & $1.12 \mathrm{e}+02$ \\
\hline \multicolumn{6}{|c|}{ Actinides } \\
\hline $\begin{array}{l}u-234 \\
U-235 \\
u-236 \\
U-238 \\
\end{array}$ & $\begin{array}{l}3.92 \mathrm{e}-01 \\
1.31 \mathrm{e}-02 \\
7.12 \mathrm{e}-02 \\
3.35 \mathrm{e}-01 \\
\end{array}$ & $\begin{array}{r}1.11 e-02 \\
3.56 e-04 \\
1.89 e-03 \\
8.35 e-03 \\
\end{array}$ & $\begin{array}{l}\text { Pu-241 } \\
\text { Pu-242 } \\
\text { Am-241 } \\
\text { An-242 } \\
\end{array}$ & $\begin{array}{l}9.42 \mathrm{e}+03 \\
7.46 \mathrm{e}-02 \\
2.92 \mathrm{e}+02 \\
3.19 \mathrm{e}-01 \\
\end{array}$ & $\begin{array}{l}2.92 \mathrm{e}-01 \\
2.16 \mathrm{e}-03 \\
9.57 \mathrm{e}+00 \\
2.66 \mathrm{e}-03\end{array}$ \\
\hline $\begin{array}{l}\text { Hp-237 } \\
\text { Pu-238 } \\
\text { Pu-239 } \\
\text { Pu-240 } \\
\end{array}$ & $\begin{array}{r}4.42 \mathrm{e}-02 \\
1.28 \mathrm{e}+02 \\
1.68 \mathrm{e}+02 \\
1.28 \mathrm{e}+02 \\
\end{array}$ & $\begin{array}{l}1.27 \mathrm{e}-03 \\
4.16 \mathrm{e}+00 \\
5.14 \mathrm{e}+00 \\
3.90 \mathrm{e}+00 \\
\end{array}$ & $\begin{array}{l}\mathrm{Am}-242 \mathrm{~m} \\
\mathrm{Am}-243 \\
\mathrm{Cm}-242 \\
\mathrm{Cm}-244 \\
\end{array}$ & $\begin{array}{l}3.21 \mathrm{e}-01 \\
2.22 \mathrm{e}-01 \\
2.65 \mathrm{e}-01 \\
4.62 \mathrm{e}+00 \\
\end{array}$ & $\begin{array}{r}4.91 \mathrm{e}-04 \\
7.02 \mathrm{e}-03 \\
9.59 \mathrm{e}-03 \\
1.59 \mathrm{e}-01 \\
\end{array}$ \\
\hline \multicolumn{4}{|c|}{ Actinide Totals } & $1.01 \mathrm{e}+04$ & $2.33 \mathrm{e}+01$ \\
\hline
\end{tabular}

06/02/1997 RADNUC2A run for shielding design basis (Mik IV fuel at 16X Pu-240). Results decayed to $05 / 31 / 1998$, Total mass of fuel in this run $2.60 \mathrm{e}+00$ MTU. Total activity of fuel in this run $1.32 e+05$ ci. Total heat generation from the fuel in this run $3.55 \mathrm{e}+02 \mathrm{~W}$. 


\section{Table 4-12. N Fuel Source Terms Continued}

Safety/Regulatory Assessment Design Basis Feed

Based on MK IV Fuel at $16.72 \%$ Pu-240.

\begin{tabular}{|l|l|} 
SAFETY BASIS VALUE Endiote No. & DESIGN BASIS VALUE Endrote No. \\
\hline Safety Basis and Design Basis are the same.
\end{tabular}

\begin{tabular}{|c|c|c|c|c|c|}
\hline I sotope & $\begin{array}{l}\text { Activity } \\
\text { (Ci/MTU) }\end{array}$ & $\begin{array}{l}\text { Heat } \\
\text { Generation } \\
\text { (w/Mru) }\end{array}$ & Isotope & $\begin{array}{l}\text { Activity } \\
\text { (Ci/MTU) }\end{array}$ & $\begin{array}{l}\text { Heat } \\
\text { Generation } \\
\text { (W/MTU) }\end{array}$ \\
\hline \multicolumn{6}{|c|}{ Fission and Activation Products } \\
\hline $\begin{array}{l}H-3 \\
C-14 \\
F e-55 \\
C 0-60\end{array}$ & $\begin{array}{l}2.61 \mathrm{e}+01 \\
5.53 \mathrm{e}-01 \\
5.41 \mathrm{e}-01 \\
2.09 \mathrm{e}+00 \\
\end{array}$ & $\begin{array}{l}8.77 e-04 \\
1.62 e-04 \\
1.80 e-05 \\
3.23 e-02 \\
\end{array}$ & $\begin{array}{l}\mathrm{s} n-123 \\
\mathrm{Sn}-126 \\
\mathrm{sb}-124 \\
\mathrm{sb}-125 \\
\end{array}$ & $\begin{array}{r}1.72 \mathrm{e}-16 \\
1.29 \mathrm{e}-01 \\
0.00 \\
0.00 \\
\end{array}$ & $\begin{array}{r}5.35 e-19 \\
3.97 e-05 \\
0.00 \\
0.00 \\
\end{array}$ \\
\hline $\begin{array}{l}\mathrm{Ni}-59 \\
\mathrm{Ni}=63 \\
\mathrm{Se}-79 \\
\mathrm{Kr}=85 \\
\end{array}$ & $\begin{array}{l}3.18 \mathrm{e}-02 \\
3.47 \mathrm{e}+00 \\
6.54 \mathrm{e}-02 \\
3.70 \mathrm{e}+02 \\
\end{array}$ & $\begin{array}{l}1.26 \mathrm{e}-06 \\
3.51 \mathrm{e}-04 \\
2.02 \mathrm{e}-05 \\
5.53 \mathrm{e}-01 \\
\end{array}$ & $\begin{array}{l}S b-126 \\
S b-126 m \\
T e-123 m \\
T e-125 m \\
\end{array}$ & $\begin{array}{r}1.81 \mathrm{e}-02 \\
1.29 \mathrm{e}-01 \\
1.50 \mathrm{e}-21 \\
0.00 \\
\end{array}$ & $\begin{array}{r}3.28 \mathrm{e}-04 \\
1.66 \mathrm{e}-03 \\
2.18 \mathrm{e}-24 \\
0.00 \\
\end{array}$ \\
\hline $\begin{array}{l}S r-89 \\
S r-90 \\
y=90 \\
y-91 \\
\end{array}$ & $\begin{array}{r}0.00 \\
6.93 \mathrm{e}+03 \\
6.93 \mathrm{e}+03 \\
0.00 \\
\end{array}$ & $\begin{array}{r}0.00 \\
8.03 \mathrm{e}+00 \\
3.84 \mathrm{e}+01 \\
0.00 \\
\end{array}$ & $\begin{array}{l}\text { Te-127 } \\
\text { Te-127m } \\
T e-129 \\
T e-129 \mathrm{~m} \\
\end{array}$ & $\begin{array}{r}2.12 e-19 \\
2.16 e-19 \\
0.00 \\
0.00 \\
\end{array}$ & $\begin{array}{r}2.85 \mathrm{e}-22 \\
1.07 \mathrm{e}-22 \\
0.00 \\
0.00 \\
\end{array}$ \\
\hline $\begin{array}{l}\mathrm{Zr}-93 \\
\mathrm{zr}-95 \\
\mathrm{Hb}-93 \mathrm{~m} \\
\mathrm{Hb}-95\end{array}$ & $\begin{array}{r}2.95 e-01 \\
0.00 \\
1.93 e-01 \\
0.00 \\
\end{array}$ & $\begin{array}{r}3.40 \mathrm{e}-05 \\
0.00 \\
3.45 \mathrm{e}-05 \\
0.00 \\
\end{array}$ & $\begin{array}{l}I-129 \\
C s-134 \\
C s-135 \\
C s-137 \\
\end{array}$ & $\begin{array}{l}5.16 \mathrm{e}-03 \\
6.47 \mathrm{e}+00 \\
6.04 \mathrm{e}-02 \\
9.66 \mathrm{e}+03 \\
\end{array}$ & $\begin{array}{l}2.41 \mathrm{e}-06 \\
6.59 \mathrm{e}-02 \\
2.02 \mathrm{e}-05 \\
9.78 \mathrm{e}+00 \\
\end{array}$ \\
\hline $\begin{array}{l}\mathrm{Nb}-95 \mathrm{~m} \\
7 c-99 \\
R u-103 \\
\mathrm{Ru}-106 \\
\end{array}$ & $\begin{array}{r}0.00 \\
2.19 \mathrm{e}+00 \\
0.00 \\
2.56 \mathrm{e}-02 \\
\end{array}$ & $\begin{array}{r}0.00 \\
1.10 e-03 \\
0.00 \\
1.52 e-06 \\
\end{array}$ & $\begin{array}{l}\text { Ba-137m } \\
\text { Ce-141 } \\
\text { Ce-144 } \\
\text { Pr- }-143 \\
\end{array}$ & $\begin{array}{r}9.14 \mathrm{e}+03 \\
0.00 \\
7.91 \mathrm{e}-04 \\
0.00 \\
\end{array}$ & $\begin{array}{r}3.59 \mathrm{e}+09 \\
0.00 \\
5.23 \mathrm{e}-07 \\
0.00 \\
\end{array}$ \\
\hline $\begin{array}{l}R h-103 m \\
R h-106 \\
P d-107 \\
\mathrm{Ag}-110 \\
\end{array}$ & $\begin{array}{r}0.00 \\
2.56 \mathrm{e}-02 \\
1.56 \mathrm{e}-02 \\
7.17 \mathrm{e}-10 \\
\end{array}$ & $\begin{array}{r}0.00 \\
2.45 e-04 \\
8.56 e-07 \\
5.38 \mathrm{e}-15 \\
\end{array}$ & $\begin{array}{l}\text { Pr- } 144 \\
\text { Pr- } 144 m \\
\text { Pm-147 } \\
\text { Pm- } 148 \\
\end{array}$ & $\begin{array}{r}7.82 \mathrm{e}-04 \\
9.48 \mathrm{e}-06 \\
1.09 \mathrm{e}+02 \\
0.00 \\
\end{array}$ & $\begin{array}{r}5.73 e-06 \\
3.18 \mathrm{e}-09 \\
4.02 \mathrm{e}-02 \\
0.00 \\
\end{array}$ \\
\hline $\begin{array}{l}\mathrm{Ag}-110 \mathrm{~m} \\
\mathrm{Cd}-113 \mathrm{~m} \\
\mathrm{~cd}-115 \mathrm{~m} \\
\mathrm{In}-113 \mathrm{~m} \\
\end{array}$ & $\begin{array}{r}5.39 e-08 \\
2.78 e+00 \\
0.00 \\
1.36 e-19 \\
\end{array}$ & $\begin{array}{r}8.94 \mathrm{e}-10 \\
3.06 \mathrm{e}-03 \\
0.00 \\
3.13 \mathrm{e}-22 \\
\end{array}$ & $\begin{array}{l}P m-148 \pi \\
S m-151 \\
E u-152 \\
E u-154 \\
\end{array}$ & $\begin{array}{r}0.00 \\
1.02 \mathrm{e}+02 \\
8.45 \mathrm{e}-01 \\
1.13 \mathrm{e}+02 \\
\end{array}$ & $\begin{array}{r}0.00 \\
1.19 e^{-02} \\
3.81 \mathrm{e}-03 \\
1.01 \mathrm{e}+00 \\
\end{array}$ \\
\hline $\begin{array}{l}5 n-113 \\
5 n-119 m \\
5 n-121 m \\
\end{array}$ & $\begin{array}{l}1.36 \mathrm{e}-19 \\
6.14 \mathrm{e}-08 \\
6.27 \mathrm{e}-02 \\
\end{array}$ & $\begin{array}{l}2.26 \mathrm{e}-23 \\
3.16 \mathrm{e}-11 \\
6.59 \mathrm{e}-05 \\
\end{array}$ & $\begin{array}{l}E u-155 \\
G d-153 \\
T b-160 \\
\end{array}$ & $\begin{array}{r}1.06 \mathrm{e}+01 \\
5.19 \mathrm{e}-10 \\
0.00 \\
\end{array}$ & $\begin{array}{r}7.65 \mathrm{e}-03 \\
4.67 \mathrm{e}-13 \\
0.00 \\
\end{array}$ \\
\hline \multicolumn{4}{|c|}{ Fission and Activation Product Totals } & $3.34 \mathrm{e}+04$ & $9.38 \mathrm{e}+01$ \\
\hline \multicolumn{6}{|c|}{ Actinides } \\
\hline $\begin{array}{l}U-234 \\
U-235 \\
U-236 \\
U-238 \\
\end{array}$ & $\begin{array}{l}3.84 \mathrm{e}-01 \\
1.27 \mathrm{e}-02 \\
7.16 \mathrm{e}-02 \\
3.31 \mathrm{e}-01 \\
\end{array}$ & $\begin{array}{l}1.09 \mathrm{e}-02 \\
3.44 \mathrm{e}-04 \\
1.91 \mathrm{e}-03 \\
8.23 \mathrm{e}-03\end{array}$ & $\begin{array}{l}\text { Pu-241 } \\
P u-242 \\
\text { An- } 241 \\
\text { An-242 } \\
\end{array}$ & $\begin{array}{l}6.82 \mathrm{e}+03 \\
8.71 \mathrm{e}-02 \\
4.34 \mathrm{e}+02 \\
3.71 \mathrm{e}-01\end{array}$ & $\begin{array}{l}2.11 \mathrm{e}-01 \\
2.55 \mathrm{e}-03 \\
1.42 \mathrm{e}+01 \\
3.08 \mathrm{e}-03\end{array}$ \\
\hline $\begin{array}{l}\text { Np-237 } \\
\text { Pu-238 } \\
P u-239 \\
\text { Pu-240 }\end{array}$ & $\begin{array}{l}4.66 \mathrm{e}-02 \\
1.33 \mathrm{e}+02 \\
1.73 \mathrm{e}+02 \\
1.37 \mathrm{e}+02 \\
\end{array}$ & $\begin{array}{l}1.34 \mathrm{e}-03 \\
4.34 \mathrm{e}+00 \\
5.28 \mathrm{e}+00 \\
4.19 \mathrm{e}+00 \\
\end{array}$ & $\begin{array}{l}A m-242 m \\
A m-243 \\
C m-242 \\
C m-244 \\
\end{array}$ & $\begin{array}{l}3.72 \mathrm{e}-01 \\
2.78 \mathrm{e}-01 \\
3.08 \mathrm{e}-01 \\
4.47 \mathrm{e}+00 \\
\end{array}$ & $\begin{array}{l}5.71 \mathrm{e}-04 \\
8.82 \mathrm{e}-03 \\
1.11 \mathrm{e}-02 \\
1.54 \mathrm{e}-01 \\
\end{array}$ \\
\hline \multicolumn{4}{|c|}{ Actinide Totals } & $7.70 \mathrm{e}+03$ & $2.85 e+01$ \\
\hline
\end{tabular}

06/02/1997 RAONUC2A run for the safety basis (Mk IV fuel at 16.72\% Pu-240). Results decayed to 05/31/1998. Total mass of the fuel in this run 1.16e+0t HTU. Total activitu of the fuel in this run $4.77 \mathrm{e}+05 \mathrm{ci}$. Total heat generation of the fuel in this run $1.42 \mathrm{e}+03 \mathrm{~W}$. 


\section{Table 4-12. N Fuel Source Terms Continued}

Safety/Regulatory Assessment Basis

Decayed to $5 / 31 / 2038$.

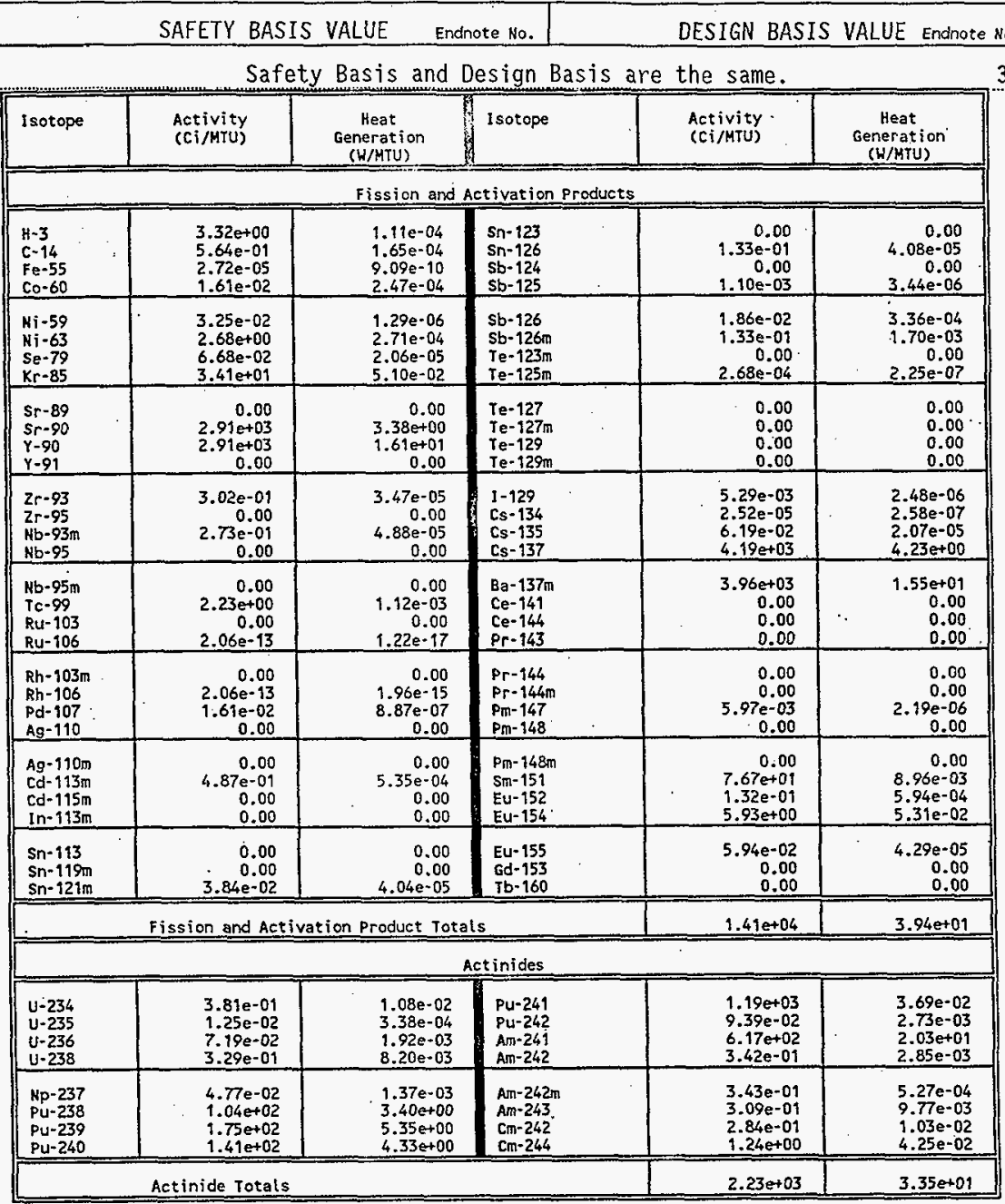

06/04/1997 RADNUC2A run for safety basis fuel (following 40 years CSB storage). Results decayed to $05 / 31 / 2038$. Total mass of fuel in this run $6.90 \mathrm{e}+00$ MTU. Total activity of the fuel in this run $1.13 \mathrm{e}+05 \mathrm{Ci}$. Total heat generation from the fuel in this run $5.04 \mathrm{e}+02 \mathrm{~W}$. 
Table 4-13. Single Pass Reactor Source Terms

Total Activity of Selected Radionuclides in SPR FueT Decayed to $1 / 1 / 94$.

SAFETY BASIS VALUE Endnote No. DESIGN BASIS VALUE Endnote No.

Safety Basis and Design Basis are the same.

Radionuclide

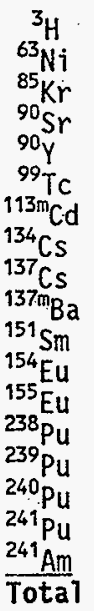

Activity (Ci)

$7.03 \mathrm{e}+02$

$6.76 \mathrm{e}+01$

$7.33 e+02$

$1.36 e+04$

1. $36 \mathrm{e}+04$

$6.67 \mathrm{e}+00$

$1.69 e+01$

6. $76 e+00$

3. $48 \mathrm{e}+04$

$3.30 \mathrm{e}+04$

4. $06 \mathrm{e}+02$

$4.70 \mathrm{e}+02$

$1.15 \mathrm{e}+02$

$1.45 e+02$

$4.06 \mathrm{e}+02$

$6.42 \mathrm{e}+02$

$1.69 \mathrm{e}+04$

$1.45 e+03$

$7.05 e+04$ 
Table 4-13. Single Pass Reactor Fuel Source Terms Continued

Total Chemical Inventory in SPR Fuel Metals

All quantities in $\mathrm{kg}$.

SAFETY BASIS VALUE Endinote No.

DESIGN BASIS VALUE Endnote No.

Safety Basis and Design Basis are the same.

Na indicates that the impurity is not present at an appreciable level.

Uranium

Impurity

Boron

Cadmium

Carbon

Chromium

Cobalt

Copper

Iron

Lithium

Magnesium

Manganese

Nickel

Nitrogen

Silicon

Others, each

Others, total
A170y 501

NA ${ }^{1}$

NA

$2.54 \mathrm{e}+00$

2. $20 \mathrm{e}-01$

NA

NA

5.07e-01

NA

$8.45 \mathrm{e}-02$

$8.45 \mathrm{e}-02$

3. $38 \mathrm{e}-01$

$3.38 \mathrm{e}-01$

2.54e-01

NA

NA
Aluminum

AlToy $X-8001$ A7-Si Braze

$1.20 \mathrm{e}-03$

3. $60 \mathrm{e}-03$

NA

NA

$1.20 \mathrm{e}-03$

$1.77 \mathrm{e}-01$

$8.40 \mathrm{e}-01$

$9.60 \mathrm{e}-03$

NA

NA

$1.40 \mathrm{e}+00$

NA

2.05e-01

$6.00 e-02$

$1.80 \mathrm{e}-01$
NA

NA

NA

NA

NA

9. $30 \mathrm{e}-03$

3. $00 \mathrm{e}-02$

NA

NA

NA

NA

NA

NA

$3.00 \mathrm{e}-03$

$1.20 \mathrm{e}-02$ 
Table 4-14. Thermal Emissivity

THERMAL EMISSIVITY

Conservatively Low Heat

Transfer Case

\begin{tabular}{|c|c|c|c|c|c|}
\hline & & SAFETY BASIS VALUE & Endrote Ho. & DESIGN BASIS VALUE & note No. \\
\hline $\begin{array}{l}\text { Stainless } \\
\text { Steel }\end{array}$ & 0.30 & 37 & & NDA & NDA \\
\hline Oxidized Fuei & 0.70 & 38 & & MDA & NDA \\
\hline ransfer Case & NDA & NDA & & NDA & NDA \\
\hline
\end{tabular}

Conservatively High Heat Transfer Case

Table 4-15. CSB́ Annual Temperature Cycle

CANISTER STORAGE BUILDING (CSB) ANNUAL TEMPERATURE CYCLE

\begin{tabular}{|l|c|l|l|}
\hline \multicolumn{2}{|c|}{ SAFETY BASIS VALUE } & OESIGN BASIS VALUE & Endrote Ho. \\
\hline $\begin{array}{l}\text { Historical Monthly Maxima and Minima for } \\
\text { Hanford Site }\end{array}$ & 39 & $\begin{array}{l}\text { Historical Monthly Averages for } \\
\text { Hanford Site }\end{array}$ & 40 \\
\hline
\end{tabular}




$$
\text { HNF-SD-SNF-TI-015, Revision } 5
$$

\subsection{DERIVED PHYSICAL QUANTITIES (calculated values)}

Section 5.0 provides a set of derived physical quantities whose values are products of key engineering, physical, and chemical parameter values in specific process scenarios.

The values are provided as input guidance for design and safety calculations, and arc. considered maximum calculated values using the appropriate safety basis, assumptions, worst case MCO properties and operating conditions relative to the selected physical quantity. These values do not address accident cases. Care should be taken, as they are not key parameter values, but derived variables and should be used within like processing scenarios only.

Table 5-1. Particulate Mass Generated per MCO

\begin{tabular}{|c|c|c|c|c|}
\hline \multicolumn{5}{|c|}{ PARTICULATE MASS GENERATED per MCO (Uranium Metal) } \\
\hline & \multicolumn{2}{|c|}{ Calculated Safety Basis } & \multicolumn{2}{|c|}{$\begin{array}{c}\text { Calculated Design Basis } \\
\text { Value Endnote }\end{array}$} \\
\hline $\begin{array}{l}\text { Between Fuel } \\
\text { Washing at basins } \\
\text { and Bulk Water } \\
\text { Draining at CVD } \\
\text { Facility }\end{array}$ & $\begin{array}{l}13 \mathrm{~kg} \text { Uranium } \\
\left(15 \mathrm{~kg} \text { as } \mathrm{UO}_{2}\right)\end{array}$ & 26 & $\begin{array}{l}66.0 \mathrm{~g} \\
\text { (Equivalent to } 75 \mathrm{~g} \text { of } \mathrm{UO}_{2} \text { ) }\end{array}$ & 18 \\
\hline $\begin{array}{l}\text { Between Bulk } \\
\text { Water Draining at } \\
\text { CVD Facility and } \\
\text { Receipt at CSB }\end{array}$ & TBD & TBD & TBD & TBD \\
\hline $\begin{array}{l}\text { During } 40 \text { Years } \\
\text { of Interim } \\
\text { Storage at CSB }\end{array}$ & TBD & TBD & $\mathrm{TBD}$ & TBD \\
\hline Total Generated & TBD & TBD & TBD & TBD \\
\hline
\end{tabular}


Table 5-2. MCO Maximum Internal Pressure

\begin{tabular}{|c|c|c|}
\hline \multicolumn{3}{|c|}{ MCO MAXIMUM INTERNAL PRESSURE } \\
\hline & \multicolumn{2}{|c|}{ Calculated Safety Basis VaTue } \\
\hline & & Endnote \\
\hline MCO Maximum Internal Pressure & $\begin{array}{r}4.2 \mathrm{~atm} \text { absolute } \\
(61.7 \text { psia })\end{array}$ & 47 \\
\hline
\end{tabular}

Tab1 35 3. MCO Maximum Temperatures in CSB Storage

\begin{tabular}{|c|c|c|c|}
\hline \multicolumn{4}{|c|}{ MCO MAXIMUM TEMPERATURES In CSB STORAGE } \\
\hline \multirow{2}{*}{$\begin{array}{l}\text { Assuming a } 7 \text { day storage with vault } \\
\text { temperature at } 62{ }^{\circ} \mathrm{C} \text { implying a bounding } \\
\text { CSB storage tube temperature of } 92{ }^{\circ} \mathrm{C} \text {. }\end{array}$} & \multicolumn{3}{|c|}{ Calculated Safety Basis Value } \\
\hline & & & Endnote \\
\hline MCO Maximum Wall Temperature & $108^{\circ} \mathrm{C}$ & $\left(226^{\circ} \mathrm{F}\right)$ & 48 \\
\hline MCO Maximum Gas Temperature & $125^{\circ} \mathrm{C}$ & $\left(257^{\circ} \mathrm{F}\right)$ & 49 \\
\hline MCO Maximum Fuel Temperature & $153^{\circ} \mathrm{C}$ & $\left(307^{\circ} \mathrm{F}\right)$ & 50 \\
\hline MCO Maximum Scrap Temperature & $123^{\circ} \mathrm{C}$ & $\left(253^{\circ} \mathrm{F}\right)$ & 57 \\
\hline
\end{tabular}

Table 5-4. Water Content Associated with Particulate in MCO

\begin{tabular}{|c|c|}
\hline \multicolumn{2}{|c|}{ WATER CONTENT ASSOCIATED WITH PARTICULATE IN MCO } \\
\hline & $\begin{array}{r}\text { Calculated Safety Basis Value } \\
\text { Endnote }\end{array}$ \\
\hline $\begin{array}{l}\text { Less than } 10^{-6} \text { Probability of Water } \\
\text { Content Associated with Uranium Oxide } \\
\text { Particulate in MCO }\end{array}$ & $.65 \mathrm{~kg}$ \\
\hline
\end{tabular}




\section{HNF-SD-SNF-TI-015, Revision 5}

\subsection{ENDNOTES}

The endnote sources identified in Section 4 and Section 5 are listed numerica7ly. Table 6-1 correlates each endnote source document with a status of the review processes required and performed in support of the office of Civilian Radioactive Waste Management (OCRWM) Quality Assurance Requirements and Description (QARD), DOE/RW/0333P.

1 D. E. Ball and D. R. Duncan, "Fuel Surface Area," HNF-SD-SNF-CN-017, Revision 2 , released June 5, 1998, p. 10.

2 HNF-SD-SNF-CN-017, Revision 2, released June 5, 1998, p. 7.

3-4 HNF-SD-SNF-CN-017, Revision 2, released June 5, 1998, p. 9.

5 T. D. Cooper and A. L. Pajunen, "SNF Project Recommended Reaction Rate Constants for Corrosion of N-Reactor Fue T, " HNF-SD-SNF-TI-020, Revision 2, released June 15, 1998, p. 4-2.

6- 8 HNF-SD-SNF-TI-020, Revision 2, released June 15, 1998, p. 2-1..

9 D. L. Sherre11, "Spent Nuclear Fuel Inventory in Bulk MCO Water at the Cold Vacuum Drying Facility," HNF-SD-W441-CN-001, Revision 2, released June 11,1998, p. 3, Table 2-1.

10-12 A. L. Pajunen, "Evaluation of Radiolytic Gas Generation from Water Dissociation in a Multi-Canister Overpack," HNF-SD-SNF-CN-006, Revision 0, released April 21, 199 7, p. 10, Table 1.

13 HNF-SD-SNF-CN-017, Revision 2, released June 5, 1998, p. 7.

14 HNF-SD-SNF-CN-017, Revision 2, released June 5, 1998, p. 6.

"Average value of $A_{g}=1.7 \times 10^{3} \mathrm{~cm}^{2}$ per $M C O$, assuming 4 fuel baskets per MCO."

$$
1,700 \mathrm{~cm}^{2} / 4 \text { baskets }=425 \mathrm{~cm}^{2} \text { per basket }
$$

15 D. R. Duncan and D. E. Ball, "K-Basins Particulate Water Content, Behavior, and Impact," HNF-1523, Revision 0, released November 19, 1997, p. 4. Table 2-2, Bounding.

16 A. B. Johnson, Jr. and A. L. Pitner, "Surface Area Considerations for Corroding N Reactor Fuel," PNNL-11174, p. 19.

17. HNF-1523, Revision 0, released November 19, 1997, p. 4. Table 2-2, Best-Estimate.

18 HNF-SD-W441-CN-001, Revision 2, released June 11, 1998, p. 3, Table 2-1. 


$$
\text { HNF-SD-SNF-TI-015, Revision } 5
$$

19 A. L. Pitner, "Summary Assessment of Fuel Damage Distributions in the $\mathrm{K}$ Basins," HNF-2586, Revision 0, released April 23, 1998, p. 6.

20 HNF-SD-W441-CN-001, Revision 2, released June 11, 1998, p. 4, Table 2-2.

21 W. L. Willis and A. N. Praga, "105-K Basin Material Design Basis Feed Description for SNF Project Facilities," HNF-SD-SNF-TI-009, Revision 1, released January 9,1998, p. 31 footnote.

22 HNF-SD-SNF-TI-009, Revision 1, released January 9, 1998, p. 17. Table 3.1, Fuel Element Description Mark IV (mass of uranium - inner $7.48+$ outer $16.0)$.

23 HNF-SD-SNF-TI-009, Revision 1, released January 9, 1998, p. 17. Table 3.1, Fuel Element Description Mark IA (mass of uranium - inner $5.49+$ outer 11.1). footnote.

24 HNF-SD-SNF-TI-009, Revision 1, released January 19, 1998, p. 24,

25 A. L. Pajunen, "Development of Design Basis Capacity for SNF Project Systems," WHC-SD-SNF-TI-016, Revision OA, released Ju1y 21, 1997, p. 3.

26 HNF-SD-W441-CN-001, Revision 2, released June 11, 1998, p. 4, Table 2-2.

27 HNF-SD-SNF-TI-009, Revision 1, released January 9, 1998, p. 38, Table 4.5 .

28 F. Schmittroth and K. D. Dobbin, "Comparison of Spent Fuel Databases for K Basin," WHC-SD-SNF-ANAL-011, Revision 0, released September 12, 1996, p. 7.

"The SCATS and R-SCATS databases were compared on a physical basis and from a burnup perspective. With minor exceptions, the two databases were nearly identical in both the number of fuel pieces and the mass of uranium. The agreement holds on a key-by-key basis as well as globally.

Burnup for the SCATS database is about $4 \%$ to $5 \%$ higher than for the RSCATS database. Decay heat calculations which are dominated by ${ }^{90} \mathrm{Sr}$, ${ }^{137} \mathrm{Cs}$, and their daughters show the same relationship."

29 HNF-SD-SNF-TI-009, Revision 1, released January 9, 1998, p. 30, Table 3.8 .

30 HNF-SD-SNF-TI-009, Revision 1, released January 9, 1998, p. 25, Table 3.6. 


\section{HNF-SD-SNF-TI-015, Revision 5}

31 HNF-SD-SNF-TI-009, Revision 1, released January 9, 1998, p. 26, Table 3.7 .

32 HNF-SD-SNF-TI-009, Revision 1, released January 9, 1998, p. 27 , Table 3.4.

33 HNF-SD-SNF-TI-009, Revision 1, released January 9, 1998, p. 30, Table 3.8 .

34 HNF-SD-SNF-TI-009, Revision 1, released January 9, 1998, p. 31, Table 3.9 .

35 HNF-SD-SNF-TI-009, Revision 1, released January 9, 1998, p. 32, Table 3.10.

36 HNF-SD-SNF-TI-009, Revision 1, released January 9, 1998, p. 36, Table 4.3 .

37 Thermophysical Properties of Matter, "Thermal Radiative Properties Metallic Elements and Alloys," Volume 7, p. 1219.

38 L. Baker Jr., et al., "Determinations of the Total Emissivity of Polished and Oxidized Uranium Surfaces," 1963, Nuclear Science Engineering, Vol. 15, p. 218.

$39-40$ D. J. Hoitink and K. W. Burk, PNNL-11794, Hanford Site Climatological Data Summary 1.997, with Historical Data, published March 1998, Summary p. iii.

41 HNF-SD-SNF-TI-009, Revision 1, released January 9, 1998, p. 24, Table 3.6.

42 D. J. Trimble and T. L. Welsh, "Statistical Analysis of 0xidation Rates for $K$ Basin Fuel in Dry Air," HNF-SD-SNF-CN-035, Revision 1, released February 6, 1998, p. 11, Figure 1 .

Mean fit of dry air literature data. Transition to High

Temperature Correlation Calculated to provide continuous function.

43 C. A. Colmenares, "Oxidation Mechanisms and Catalytic Properties of the Actinides," Prog. Solid St. Chem. Vol. 15., p. 308, Figure 31.

44 The transitions between moist air correlations were developed to provide a consistent method of using equations to estimate reaction rates at conditions not specifically covered by data correlations. The transitions also produce continuous functions between correlations. To maintain continuity, the temperature for transition between correlations could not be specified as a single temperature, varying with the relative humidity. An implicit equation for $\mathrm{RH}<10 \%$ and explicit equation for $\mathrm{RH}>10 \%$ was developed based on an approximation of the saturation pressure variation with 
HNF-SD-SNF-TI-015, Revision 5

temperature. The simplified saturation pressure correlation, $\log \mathrm{P}_{\text {sat }}=$ $7.6726-2126.5 / T$ where $P_{\text {sat }}$ is in $\mathrm{kPa}$ and $T$ is in $K$, is within $10 \%$ of Handbook data in the region of interest. Based on the saturated steam correlation the transition temperature, $T_{I}$, between correlations is found by the following:

For $\mathrm{RH}<10 \%$, solve the following equation for $T$

$\log \left\{10^{(7.19-3732 / \mathrm{T})}+\mathrm{P}\left[10^{(1.6604-1630.5 \mathrm{~T})}-10^{(0.5174-1605.5 / \mathrm{T})}\right]\right\}-10.566-0.3 \log \mathrm{P}+4990 / \mathrm{T}=0$

where $P$ is the partial pressure of water.

For $10 \% \leq \mathrm{RH} \geq 100 \%$,

$T_{1}=\frac{1260}{2.233+0.3 \log (P)}$

45 - 46 Prog. Solid St. Chem. Vol. 15., p. 293.

47 J. R. Frederickson and M. G. Plys, "MCO Internal Gas Composition and Pressure During Intel'im Storage," HNF-SD-SNF-TI-040, Revision 2, released June 18,1998, p. 2-4.

48-49 D. R. Duncan and M. G. Plys, "SimuTation of Normal and Off-Normal Multi-Canister Overpack Behavior," HNF-2256, Revision 1, released July 1,1998, p. T-33.

50 HNF-2256, Revision 1, released July 1, 1998, p. 2-3.

$51 \mathrm{~J}$. P. Sloughter, et al., "Estimate of Particulate Mass in Multi-Canister Overpacks," HNF-1527, Revisión 0, released November 19, 1997, p. 27, Bounding.

52 HNF-1527, Revision 0, released November 19, 1997, p. 27, Nominat.

53 A. L. Pajunen and J. P. Sederburg, "Spent Nuclear Fuel Project Product Specification," HNF-SD-SNF-OCD-001, Revision 2, released March 28, 1998, p. 22.

54 A. L. Pajunen, "Cold Vacuum Drying Residual Free Water Test Description," HNF-1851, Revision 1, released June 10, 1998, p. 4.

55 D. R. Duncan and M. G. Plys, "Sensitivity of Probabilistic MCO Water Content Estimates to Key Assumptions, " HNF-2234, Revision 1, released March 23, 1998, p. 1-2.

56 HNF-SD-SNF-CN-017, Revision 2, released June 5, 1998, p. 10.

57 HNF-2256, Revision 1, released July 1, 1998, p. 2-3. 


\begin{tabular}{|c|c|c|c|c|c|c|}
\hline 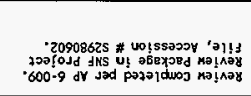 & $S \exists \lambda$ & $\mathrm{S} \exists \lambda$ & $16 / 6 \mathrm{I} / \mathrm{IT}$ & ' & 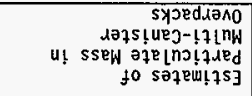 & $\begin{array}{l}0 \text { บo!s!nəy } \\
\text { 'LZS[-JNH }\end{array}$ \\
\hline 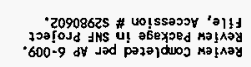 & $S \exists \lambda$ & $S \exists \lambda$ & $L 6 / 6 \mathrm{I} / \mathrm{It}$ & pue uesung ${ }^{\prime} \cdot{ }_{y}^{\prime} \cdot 0$ & 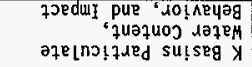 & $\begin{array}{l}0 \text { Uo!s!ควย } \\
\text { 'EZSI-JNH }\end{array}$ \\
\hline 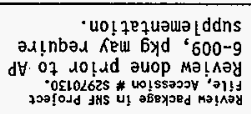 & $5 \exists \lambda$ & 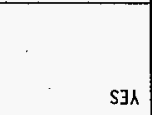 & $\angle 6 / \mathrm{tz} / \mathrm{t}$ & uəun & 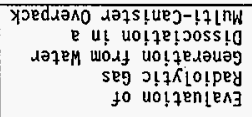 & 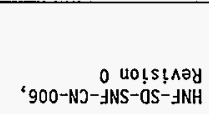 \\
\hline pazen!ena aq ol & $\begin{array}{r}\text { pazen [ena } \\
\text { əq oL }\end{array}$ & $\begin{array}{r}\text { Pazen lena } \\
\text { aq } 01\end{array}$ & $86 / \tau t / 9$ & 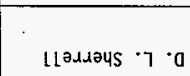 & 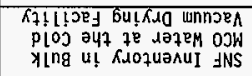 & 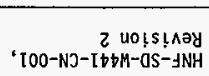 \\
\hline 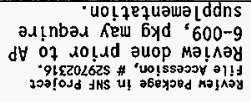 & S3X & 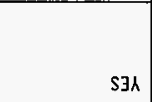 & $86 / 91 / 9$ & 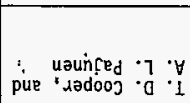 & 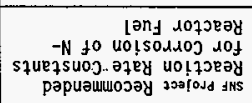 & 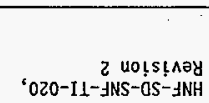 \\
\hline 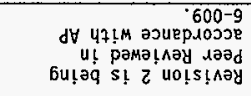 & $53 \lambda$ & $S 3 \lambda$ & $86 / 9 / 9$ & 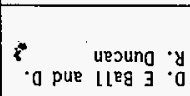 & eady әכefuns [anj & 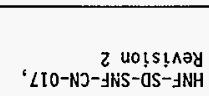 \\
\hline 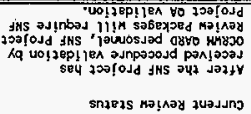 & 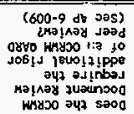 & 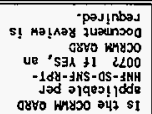 & 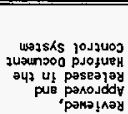 & Joụın & 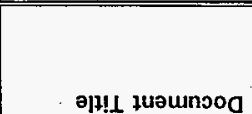 & dequnn quəunวog \\
\hline
\end{tabular}




\begin{tabular}{|c|c|c|c|c|c|c|}
\hline$\partial\lfloor q E \partial !$ Ldd $20 \mathrm{~N}$ & $\begin{array}{r}\text { alqeo! } 1 \text { Ldy } \\
\text { zON }\end{array}$ & ON & $\begin{array}{r}866 \mathrm{I} / \varepsilon \\
\text { pəus! Iqnd }\end{array}$ & 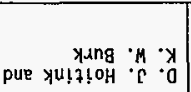 & 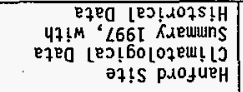 & t6LIII-7NNd \\
\hline alqeग! ldd ZON & 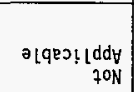 & ON & $\begin{array}{r}\varepsilon 96 \mathrm{I} \\
\text { pəus! Iqnd }\end{array}$ & 7ә & 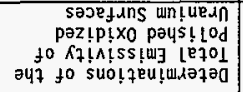 & 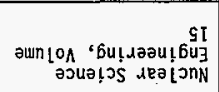 \\
\hline əLqeग! Ldd $\forall$ zON & $\begin{array}{r}\partial\lfloor q e \partial !\lfloor d d y \\
7 O N\end{array}$ & ON & pays! $\begin{array}{l}0<6 \mathrm{I} \\
19 \mathrm{n}_{\mathrm{d}}\end{array}$ & 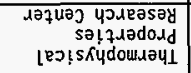 & 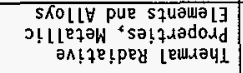 & 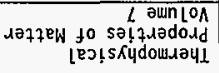 \\
\hline ә LQRo! Ldd $70 N$ & 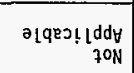 & ON & $96 / 2 ! / 6$ & 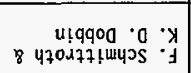 & $\begin{array}{r}\text { suiseg } \\
\text { y loy sasegezed iany } \\
\text { zuads to uos lueduoj }\end{array}$ & 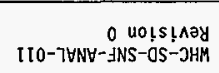 \\
\hline 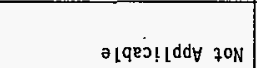 & 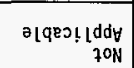 & ON & $\angle 6 / 12 / L$ & uəunçed $\left.{ }^{\prime}\right\rceil \cdot \forall$ & 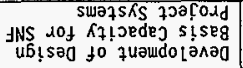 & 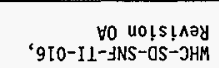 \\
\hline 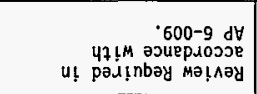 & S3h & $S \exists \curlywedge$ & $86 / 6 / I$ & 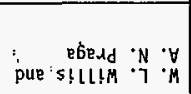 & 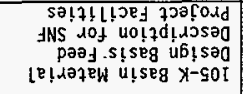 & $\begin{array}{r}\text { I Uo!s inay } \\
{ }^{6} 600-I 1-3 N S-0 S-2 N H H\end{array}$ \\
\hline 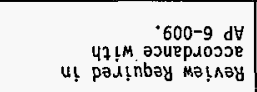 & $\mathrm{S} 3 \mathrm{\lambda}$ & $S \exists \lambda$ & $86 / \varepsilon z / b$ & גav?!d ' ך' & 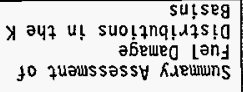 & 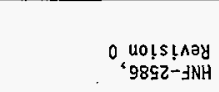 \\
\hline 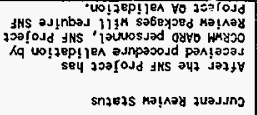 & 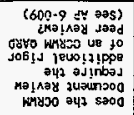 & 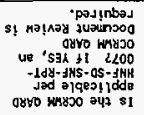 & 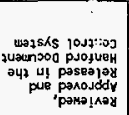 & souln & ә|!! luәunooด & dəqunh quewnכoo \\
\hline
\end{tabular}


Table 6-1. Technical Databook Supporting Document Review Status

Page 3 of 4

\begin{tabular}{|c|c|c|c|c|c|c|}
\hline Document Number & Document Title & Author & $\begin{array}{l}\text { Reviewed, } \\
\text { Approved and } \\
\text { Released in the } \\
\text { Hanford Docunent } \\
\text { Contro! Systcrat }\end{array}$ & 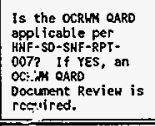 & 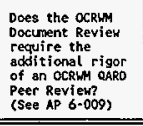 & 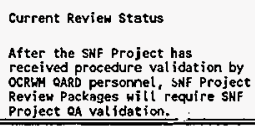 \\
\hline $\begin{array}{l}\text { HNF-SO-SNF-CN-035, } \\
\text { Revision } 1\end{array}$ & \begin{tabular}{|l|} 
Statistical Analysis of \\
Oxidation Rates for K \\
Basin Fuel in Dry Air \\
\end{tabular} & $\begin{array}{l}\text { D. J. Trimble and } \\
\text { T. L. Welsh }\end{array}$ & $2 / 6 / 98$ & YES & YES & $\begin{array}{l}\text { This docement to be revised, with } \\
\text { revien to follow. }\end{array}$ \\
\hline $\begin{array}{l}\text { Prog. Solid St. } \\
\text { Chem., Vol. } 15 .\end{array}$ & $\begin{array}{l}\text { 0xidation Mechanisms } \\
\text { and Catalytic } \\
\text { Properties of the } \\
\text { Actinides } \\
\end{array}$ & C. A. Colmenares & $\begin{array}{l}\text { Published } \\
\text { Handbook }\end{array}$ & No & $\begin{array}{l}\text { Not } \\
\text { App1 icable }\end{array}$ & Not Applicable \\
\hline $\begin{array}{l}\text { HNF-SO-SNF-TI-040, } \\
\text { Revision } 2\end{array}$ & $\begin{array}{l}\text { MCO Internal Gas } \\
\text { Composition and } \\
\text { Pressure During Interim } \\
\text { Storage }\end{array}$ & $\begin{array}{l}\text { D. R. Duncan and } \\
\text { M. G. Plys }\end{array}$ & $6 / 18 / 98$ & YES & YES & 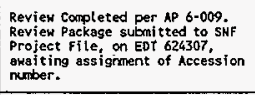 \\
\hline $\begin{array}{l}\text { HNF-2256, } \\
\text { Revision } 1\end{array}$ & $\begin{array}{l}\text { Simulation of Normal } \\
\text { and Off-Normal Multi- } \\
\text { Canister Overpack }\end{array}$ & $\begin{array}{l}\text { D. R. Duncan and } \\
\text { M. G. Plys }\end{array}$ & $7 / 1 / 98$ & YES & YES & $\begin{array}{l}\text { Review Required in } \\
\text { accordance with } \\
\text { AP 6-009. }\end{array}$ \\
\hline $\begin{array}{l}\text { HNF-2234, } \\
\text { Revision } 1\end{array}$ & $\begin{array}{l}\text { Sensitivity of } \\
\text { Probabilistic MCO Water } \\
\text { Content Estimates to } \\
\text { Key Assumptions }\end{array}$ & $\begin{array}{l}\text { D. R. Duncan and } \\
\text { M. G. Plys }\end{array}$ & $3 / 23 / 98$ & YES & YES & 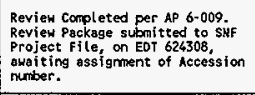 \\
\hline $\begin{array}{l}\text { HNF-SD-SNF-OCD-001, } \\
\text { Revision } 2\end{array}$ & $\begin{array}{l}\text { SNF Project Product } \\
\text { Specification }\end{array}$ & $\begin{array}{l}\text { A. L. Pajunen and } \\
\text { J. P. Sederburg }\end{array}$ & $3 / 28 / 98$ & No & NO & Not Applicable \\
\hline $\begin{array}{l}\text { HNF-1851, } \\
\text { Revision } 1\end{array}$ & $\begin{array}{l}\text { Cold Vacuum Drying } \\
\text { Residual Free Water } \\
\text { Test Description } \\
\end{array}$ & A. L. Pajunen & $6 / 10 / 98$ & YES & YES & 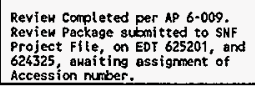 \\
\hline
\end{tabular}


HNF-SD-SNF-TI-015, Revision 5

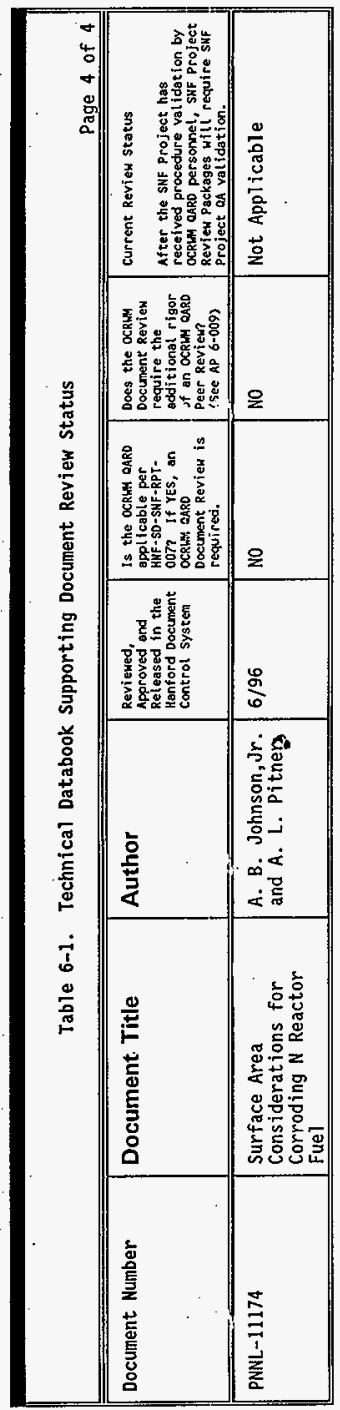




\section{DISTRIBUTION SHEET}

\begin{tabular}{|c|c|c|c|c|c|}
\hline To & \multirow{2}{*}{\multicolumn{3}{|c|}{$\begin{array}{l}\text { From } \\
\text { Process Engineering (Reilly) }\end{array}$}} & \multicolumn{2}{|c|}{ Page 1 of 1} \\
\hline SNF Project TI-015 Distribution & & & & \multicolumn{2}{|c|}{ Date July 15, 1998} \\
\hline \multicolumn{4}{|l|}{ Project Title/Work Order } & \multicolumn{2}{|c|}{ EDT No. NA } \\
\hline SNF Project Technical Databook, & HNF-SD-SNF-TI- & 15, Rev. & & \multicolumn{2}{|c|}{ ECN No. - 645109} \\
\hline Name & MSIN & $\begin{array}{c}\text { Text } \\
\text { With All } \\
\text { Attach. }\end{array}$ & Text Only & $\begin{array}{l}\text { Attach./ } \\
\text { Appendix } \\
\text { Only }\end{array}$ & $\begin{array}{l}\text { EDT/ECN } \\
\text { Only }\end{array}$ \\
\hline
\end{tabular}

\section{Central Files (orig +1 )}

SNF Project Files (54)

(The 54 copies are for controlled distribution) $\begin{array}{ll}\text { A3-88 } & X \\ \text { R3-11 } & X\end{array}$ 